\title{
Pupas Podonominae (Diptera: Chironomidae) de los ríos altoandinos tropicales
}

\author{
Raúl Acosta* \& Narcís Prat \\ Grupo de Investigación F. E. M. (Freshwater Ecology and Management), Universidad de Barcelona, Barcelona, \\ España; racosta@ub.edu,nprat@ub.edu \\ * Correspondencia
}

Recibido 20-VII-2017. Corregido 00-X-2017. Aceptado 16-XI-2017.

\begin{abstract}
Podonominae pupae (Diptera: Chironomidae) from the tropical high Andean rivers. The Podonominae is a subfamily of Chironomidae which immature stages inhabit cold streams and brooks. In South America, most of the species have been reported only from the Andean/Patagonian subregion. However, the knowledge of its diversity from high tropical areas is still scarce. We studied the richness of genera and morphotypes of pupae and pupal exuviae of Podonominae (Diptera: Chironomidae) in the high Andean rivers from Colombia, Ecuador and Peru, and discussed its geographical distribution in the studied area. The specimens were collected between 2004 and 2011 in 197 sampling points, from 17 basins over 2000 masl. During the dry season, benthic and drift samples were taken in different substrates. We recognized three genera: Podonomopsis, Parochlus and Podonomus, with one, nine and 14 morphotypes, respectively. Only six of them were clearly determined as species previously described. Podonomopsis was represented only by one species, $P$. illiesi, only found in high altitude rivers in Perú (above 3500 masl). Morphotypes richness and abundance of Parochlus were higher in rivers from Ecuador and Colombia (P. incaicus, Parochlus sp. 1 and Parochlus sp. 2 were the most common species). Conversely, Podonomus was more diverse and abundant in rivers from Perú (P. caran$q u i$, P. fittkaui and P. fastigians were the most common species). We found a clear pattern of distribution of morphotypes in the latitudinal gradient. First, we confirmed an evident decrease in the morphotypes richness in relation to the South Andes. Second, we found species such as Podomopsis illiesi, Podonomus fittkaui and Podomus fastigians that only were present in Perú, but not in Ecuador and Colombia. For these species, the Huancabamba Depression ( $6^{\circ} \mathrm{S}$ Latitude, North of Perú), could represent an important barrier in their dispersion toward lower latitudes. A taxonomic key to genera and morphotypes determination was also provided. Rev. Biol. Trop. 66(1): 6-27. Epub 2018 March 01.
\end{abstract}

Key words: Chironomidae; South America; macroinvertebrates; aquatic insects; high Andean streams; pupal exuviae.

La subfamilia Podonominae (Diptera: Chironomidae) representa un importante componente de las comunidades de macroinvertebrados en ríos y arroyos fríos de alta montaña en el hemisferio sur. Aunque esta subfamilia fue establecida originalmente a partir de especímenes recolectados de manera ocasional en ríos de la región Paleártica (Thienemann, 1937), posteriormente, con base en observaciones morfológicas detalladas de adultos, pupas, exuvias y larvas de Podonominae australes (Sudamérica, Sudáfrica, Australia y
Nueva Zelanda), Brundin (1966) realizó un extenso estudio, en el que además de revelar una sorprendente diversidad de especies en comparación con la fauna boreal, puso de relieve importantes relaciones filogenéticas y conexiones biogeográficas transantárticas (Gondwánicas) entre sus componentes. De esta manera, este trabajo de Brundin se convirtió en la principal referencia para el conocimiento de los Podonominae en Sudamérica, particularmente en los Andes del Sur (Chile y Argentina). Posteriormente, Roback (1970) describió 
dos nuevas especies del género Podonomus de los Andes de Ecuador, y Roback y Coffmann (1983) describieron tres nuevos morfotipos pupales procedentes del altiplano de Perú y Bolivia. Más recientemente, Cranston, Hardy, Morse, Puslednik y McCluen (2010), y Cranston y Kosch (2015) incorporaron evidencias moleculares que respaldaron en gran medida la filogenia propuesta originalmente por Brundin y sugirieron la presencia de especies vicariantes entre las regiones biogeográficas australes.

Sin embargo, los resultados de estos estudios se han restringido principalmente a material biológico recolectado en los Andes del Sur (al sur de Bolivia, especialmente Chile y Argentina). Por el contrario, poco se conoce con respecto a la diversidad y biogeografía de los Podonominae de la zona altoandina tropical (Andes del Norte y Centro) (Spies \& Reiss, 1996; Ashe \& O’Connor, 2009). Recientemente, Prat, González-Trujillo y Ospina-Torres (2014) publicaron una clave para la distinción de géneros y algunos morfotipos de pupas de quironómidos presentes en los ríos tropicales altoandinos desde el norte de Colombia hasta el sur de Perú. En aquél trabajo, se incluyeron los tres géneros Podonominae de amplia distribución en la zona de estudio y sobre los cuales tratará el presente trabajo: Podonomopsis Brundin, 1966; Podonomus Philippi, 1865 y Parochlus Enderlein, 1912.

Podonomopsis es un género de origen gondwánico descrito por Brundin (1966) desde los Andes de Argentina, que también cuenta con varias especies en Australia (Cranston \& Krosch, 2015). En los Andes del Sur (Argentina y Chile) cuenta con cinco especies, sin embargo, en los ríos andinos tropicales solo se presenta una especie, $P$. illiesi, descrita originalmente desde el altiplano del Titicaca en Perú. Aunque Podonomopsis puede ser localmente abundante, por lo general es poco frecuente; a diferencia de Parochlus y Podonomus, que si están muy diversificados y en Sudamérica se distribuyen a lo largo de toda la cordillera de los Andes, siendo en ocasiones los elementos que dominan la fauna bentónica, especialmente en ríos de cabecera (Brundin,
1966; Acosta \& Prat, 2010; Kuhn et al., 2011). El género Parochlus es de amplia distribución a nivel mundial y cuenta con más de 50 especies (Ashe \& O'Connor, 2009) de las cuales 29 viven en ríos de montaña de los Andes, el archipiélago del Sur de Chile y las islas Juan Fernández (Brundin, 1966). Por su parte, el género Podonomus incluye en Sudamérica 40 especies válidas y nueve morfotipos no descritos formalmente (Ashe \& O'Connor, 2009) principalmente desde los Andes de Bolivia hasta la Patagonia (Brundin, 1966). Aunque también se ha reportado una nueva especie (Roque \& TrivhinhoStrixino, 2004), y dos poblaciones adicionales genéticamente diferentes (Trivinho-Strixino, Pepinelli, Siqueira, \& Roque, 2012) en ríos del bosque lluvioso del Atlántico de Brasil entre los 1300 y $2700 \mathrm{msnm}$. Al parecer la diversidad específica de estos tres géneros de Podonominae en los ríos altoandinos tropicales, es menor a la encontrada en los Andes australes de Argentina y Chile. Por ejemplo, recientemente Cranston y Krosh (2015) revisaron morfológica y molecularmente el género Podonomopsis, e incluyeron especímenes recolectados por los autores de este artículo en la zona altoandina tropical. Los resultados mostraron que entre el sur del Perú y el norte de Colombia solo se encuentra Podonomopsis illiesi, y así se confirma lo que inicialmente sugirió Brundin (1966).

Los objetivos de este trabajo fueron: i) describir la diversidad de taxa de Podonominae en los ríos andinos tropicales entre el sur de Perú y el norte de Colombia, y por encima de los $2000 \mathrm{msnm}$, usando las características morfológicas de las exuvias pupales; y ii) comprobar si existe una evidente disminución de especies y una simplificación filogenética hacia el norte de la Cordillera de los Andes. Así mismo, con los resultados obtenidos se propone una clave para distinguir los diferentes morfotipos de pupas de Podonominae en los ríos altoandinos tropicales. Esta clave representa un complemento a la clave de géneros de pupas de ríos altoandinos de Prat et al. (2014); aunque no se han podido identificar todas las especies de los morfotipos encontrados, la elaboración de este tipo de claves representa una estrategia adecuada, 
cuando se comienza a estudiar un área con poca información taxonómica de larvas y pupas a nivel de especie. Por ejemplo, Cranston (2000), elaboró una clave de géneros y morfotipos de larvas y pupas de Chironomidae para Australia, la cual revisó constantemente y a partir de la cual se han basado posteriores estudios morfológicos y moleculares sobre grupos específicos, que han permitido la confirmación de especies originalmente descritas como morfotipos, especialmente sobre componentes de fauna gondwánica (Cranston et al., 2010; Cranston \& Sæther, 2010; Cranston \& Krosch, 2015).

\section{MATERIALES Y MÉTODOS}

El área de estudio se sitúa entre el norte de Colombia ( $5^{\circ}$ Latitud Norte) y el sur del Perú ( $16^{\circ}$ Latitud Sur) y a lo largo de un gradiente altitudinal entre los 2000 y 4600 msnm. Los especímenes de pupas y exuvias pupales provinieron de un total de 197 puntos (ríos o arroyos) distribuidos en 17 cuencas muestreadas en tres proyectos de investigación realizados entre los años 2004 a 2011 (detalles de los mismos pueden consultarse en http://www.ub.edu/ riosandes/). El Cuadro 1 muestra el número de puntos de muestreo por cuenca y por país, e información adicional puede encontrarse en el Apéndice 1. Las muestras del proyecto CERA, fueron tomadas en la temporada seca entre octubre del 2007 y noviembre del 2008, y las del proyecto BIQURA, en la temporada seca entre julio a octubre del 2011.

Los especímenes recolectados en ambos proyectos fueron obtenidos a partir de un muestreo cuantitativo realizado en transectos de 50 $\mathrm{m}$ de largo. En cada uno de ellos, fueron tomadas 12 muestras de red Surber de $0.1023 \mathrm{~m}^{2}$ y

\section{CUADRO 1}

Cuencas muestreadas para el estudio de pupas y exuvias pupales de Podonominae (Diptera: Chironomidae) en ríos altoandinos

TABLE 1

Basins sampled for the study of pupae and pupal exuviae of Podonominae (Diptera: Chironomidae) at Andean high altitude rivers

\begin{tabular}{|c|c|c|c|c|c|}
\hline País & Cuenca & Código & Vertiente & $\begin{array}{l}\text { Número } \\
\text { de sitios }\end{array}$ & Proyecto \\
\hline Colombia & Chinchiná & CHIN & Atlántico-Caribe & 6 & BIQURA \\
\hline Colombia & Campoalegre-Cauca & CAMPO & Atlántico-Caribe & 2 & BIQURA \\
\hline Colombia & Gualí-Magdalena & GUALI & Atlántico-Caribe & 2 & BIQURA \\
\hline Colombia & San Juan & JUAN & Pacífico & 2 & BIQURA \\
\hline Ecuador & Mira & MIR & Pacífico & 16 & CERA \\
\hline Ecuador & Aguarico-Napo & $\mathrm{AGU}$ & Atlántico-Amazonas & 14 & CERA \\
\hline Ecuador & Guayabamba-Esmeraldas & GUAY & Pacífico & 7 & BIQURA \\
\hline Ecuador & Papallacta, Tambo-Napo & NAPO & Atlántico-Amazonas & 6 & BIQURA \\
\hline Ecuador & Pastaza-Marañón & PAS & Atlántico-Amazonas & 16 & CERA \\
\hline Ecuador & Guayas & $\mathrm{GU}$ & Pacífico & 15 & CERA \\
\hline Perú & Santa & SAN & Pacífico & 16 & CERA,BIQURA \\
\hline Perú & Mosna-Marañón & MOS & Atlántico-Amazonas & 16 & CERA,BIQURA \\
\hline Perú & Cañete & CAÑ & Pacífico & 35 & Tesis* Ph. D. \\
\hline Perú & Urubamba & URU & Atlántico-Amazonas & 15 & CERA \\
\hline Perú & Ampay-Apurimac & AMP & Atlántico-Amazonas & 7 & BIQURA \\
\hline Perú & Cotahuasi-Ocoña & $\mathrm{COT}$ & Pacífico & 7 & BIQURA \\
\hline Perú & Colca-Camaná & $\mathrm{COL}$ & Pacífico & 15 & CERA \\
\hline
\end{tabular}

BIQURA: Biodiversidad de Quironómidos en Ríos Altoandinos (2011), CERA: Calidad Ecológica de Ríos Altoandinos (2007). Tesis* Ph. D. R. Acosta (2009). 
250 micras de apertura, de las cuales ocho pertenecieron a hábitats dominantes (más del $5 \%$ de cobertura en el transecto) y cuatro a hábitats marginales (menos del $5 \%$ de cobertura en el transecto) según su representatividad y habitabilidad en el transecto (Villamarín, Rieradevall, Paul, Barbour, \& Prat, 2013). Adicionalmente, en los puntos de muestreo del proyecto BIQURA, se realizó una separación in situ de pupas provenientes de las muestras bentónicas y se instalaron dos a tres redes de deriva durante dos a tres horas de exposición con el objetivo de recuperar pupas y exuvias pupales transportadas por la corriente (Prat, Ribera, Rieradevall, Villamarín, \& Acosta, 2013). Las muestras del río Cañete provinieron de un muestreo semicuantitativo con una red circular de mano de 250 micras de apertura, realizado durante dos ocasiones, en la temporada seca y final de la lluviosa en los años 2004 y 2005, respectivamente (Acosta \& Prat, 2010).

Las muestras bentónicas de los tres proyectos fueron conservadas en formol al $10 \%$, mientras que las muestras de deriva y los especímenes de la recolecta manual del proyecto BIQURA, se conservaron en alcohol al $96 \%$. Posteriormente, las pupas y exuvias pupales fueron separadas en el laboratorio, y montadas en láminas portaobjetos con Euparal ${ }^{\circledR}$ como medio de conservación permanente y siguiendo los protocolos descritos por Pinder y Reiss (1986) y Epler (2001), tal como se detalla en Prat et al. (2014).

La nomenclatura morfológica y la ubicación de las diferentes partes de la pupa y exuvias utilizadas en esta clave siguieron a Prat et al. (2014) (véase la fig. 1 de dicha publicación), pero se utilizó por ejemplo "Órgano Respiratorio" (OR) en lugar de "Cuerno Torácico". Para distinguir los diferentes morfotipos pupales hemos utilizado: i) el nombre científico específico (en tres casos en los que ha sido posible identificarlos a este nivel); ii) el grupo taxonómico al que pertenecen, los cuales fueron establecidos por Brundin (1966), o iii) nombrándolos como sp. 1, sp. 2 y así sucesivamente. Cuando el espécimen examinado fue muy semejante a alguna especie previamente descrita, pero la zona de recolección original era muy distante a nuestra área de estudio (por ejemplo, la región patagónica), se ha preferido designar el morfotipo como cercano o próximo a dicha especie ("nr").

\section{RESULTADOS}

De las 197 muestras tomadas se encontraron pupas o exuvias pupales de Podonominae en 73 muestras en un rango altitudinal entre los 2300 y 4800 msnm. Para todo el material examinado se observaron un total de 24 morfotipos diferentes en tres géneros (Fig. 1), cada uno de los cuales podría corresponder a una especie.

Un total de 23 individuos de Podonomopsis fueron recolectados en toda la zona de muestreo, todos pertenecientes a la especie $P$. illiesi (Brundin, 1966), y exclusivamente en las cuencas andinas de Perú por encima de los $3500 \mathrm{msnm}$. La mayor cantidad de pupas fueron recuperadas de la cuenca del río Cañete (16), pero también en los ríos Cotahuasi (5) y Santa (4).

Del género Parochlus, se examinaron 64 individuos e identificaron nueve morfotipos (Cuadro 2, Fig. 2 y Fig. 3). Todos los especímenes recolectados (excepto Parochlus nigrinus peruvianus) tienen sedas largas y onduladas en el segmento anal (dos sedas) y en el segmento abdominal VIII (cinco sedas). Las espuelas anales (AS) nunca son muy grandes (máximo de 67 micras) y todos tienen el abdomen con una microescultura poco desarrollada, con lo cual se descarta la presencia de especies del grupo patagonicus, que sí presentan una microescultura evidente. Por otra parte, las AS nunca están en ángulo recto hacia fuera, ni tienen fuertes dentículos, por lo tanto, los grupos squamipalpis y steineni tampoco parecen encontrarse en la zona altoandina tropical. Por lo que todas las especies encontradas en nuestro estudio se sitúan dentro de los grupos nigrinus y araucanus.

Dentro del primer grupo, destaca P. nigrinus con la subespecie peruvianus descrita previamente por Brundin (1966) (Fig. 2A). Las especies de este grupo se distinguen por 

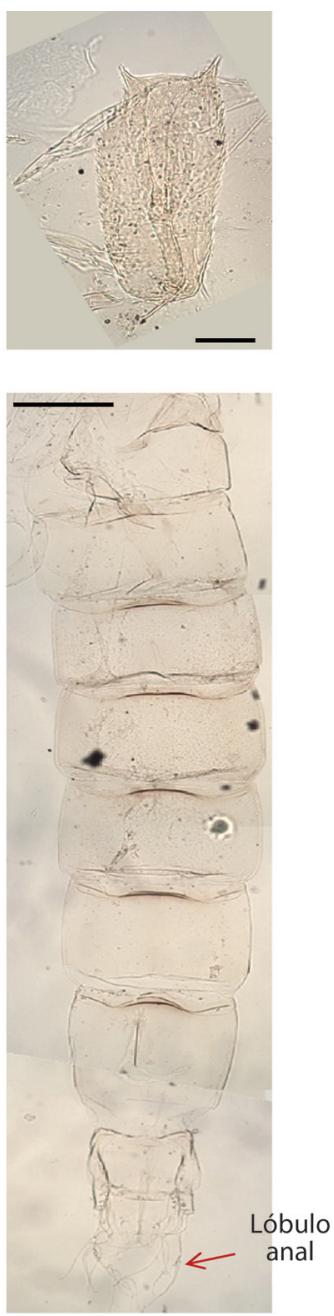

A
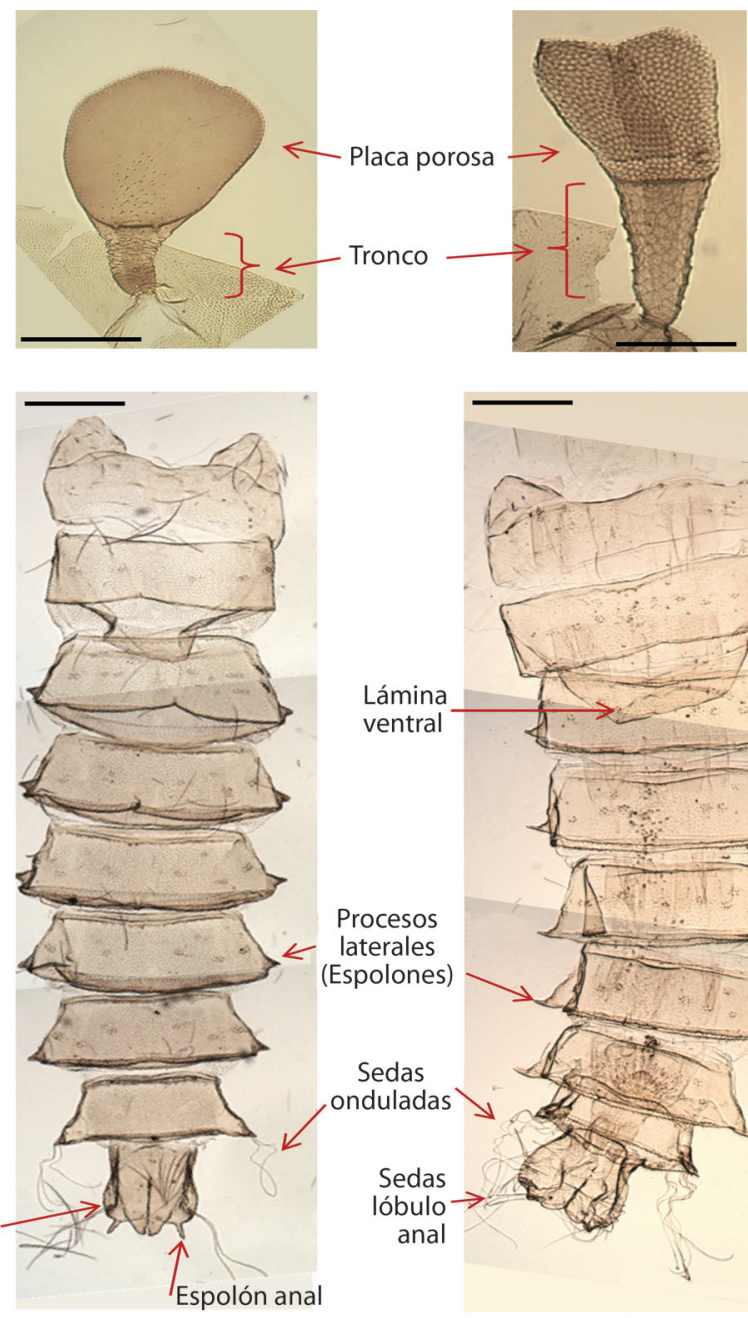

B

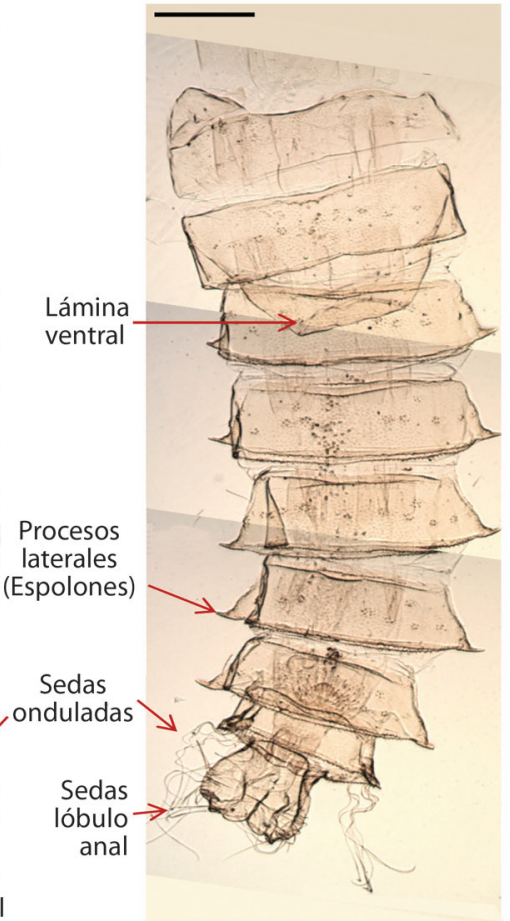

C

Fig. 1. Características morfológicas usadas en la clave para distinguir los géneros, grupos de especies y morfotipos de Podonominae (pupas y exuvias pupales) en ríos altoandinos. Los órganos respiratorios (OR) y el abdomen son mostrados en la parte superior e inferior, respectivamente, A) Podonomopsis B) Parochlus y C) Podonomus. Líneas de escala: OR (A: $25 \mu \mathrm{m}$, B y C: $50 \mu \mathrm{m})$; Abdomen (A, B y C: $100 \mu \mathrm{m})$.

Fig. 1. Morphological characteristics used in the key to recognize genera, groups of species and morphotypes of Podonominae (pupae and pupal exuviae) at the Andean high altitude rivers. The breathe organs (thoracic horns) and the abdomen are showed in the upper and lower part, respectively, A) Podonomopsis B) Parochlus and C) Podonomus. Bar scale: OR (A: $25 \mu \mathrm{m}$, B y C: $50 \mu \mathrm{m}$ ); Abdomen (A, B y C: $100 \mu \mathrm{m}$ ).

la ausencia de sedas onduladas largas tanto en el segmento anal como en el segmento abdominal VIII; en nuestros muestreos fue recolectado un solo individuo en la cuenca del río Colca. Los otros ocho morfotipos pertenecieron al grupo araucanus, que por la forma del órgano respiratorio (OR) se presentaron dos subgrupos: araucanus, con la longitud de la placa porosa (PP) relativamente pequeña con respecto al tronco $(\mathrm{T})$ (Relación LT/LPP $>0.5$ ), y trigonocerus con la PP relativamente grande con respecto al $\mathrm{T}$ (Relación LT/LPP $<0.5)$. Dentro de este último subgrupo, tanto Parochlus sp. 2 (Fig. 2D) como P. incaicus 
CUADRO 2

Riqueza y abundancia de especies y morfotipos de pupas y exuvias pupales de Parochlus recolectados en ríos altoandinos

TABLE 2

Richness and abundance of species and morphotypes of pupae and pupal exuviae of Parochlus collected at Andean high altitude rivers

\begin{tabular}{|c|c|c|c|c|c|c|c|c|c|c|c|}
\hline & & CHIN $^{1}$ & $\mathrm{MIR}^{2}$ & $\mathrm{NAP}^{2}$ & GUAY $^{2}$ & SAN $^{3}$ & $\operatorname{MOS}^{3}$ & CAÑ $\tilde{N}^{3}$ & $\mathrm{COL}^{3}$ & $\mathrm{URU}^{3}$ & Total \\
\hline \multicolumn{12}{|l|}{ Grupo araucanus } \\
\hline \multirow[t]{4}{*}{ Sub-grupo araucanus } & P. nr. araucanus & & & & & & & 1 & & & 1 \\
\hline & Parochlus sp. 1 & & & 2 & 6 & 6 & 3 & & & & 17 \\
\hline & Parochlus sp. 3 & 1 & & & & & & & & & 1 \\
\hline & P. nr. maorii & & & & 1 & & & & & & 1 \\
\hline \multirow[t]{4}{*}{ Sub-grupo trigonocerus } & P. nr. ohakunensis & 6 & 2 & & 1 & & & & & & 9 \\
\hline & P. incaicus & & & 5 & & 3 & & 6 & & 2 & 16 \\
\hline & Parochlus sp. 2 & 9 & 3 & & 5 & & & & & & 17 \\
\hline & Parochlus sp. 4 & & & & & 1 & & & & & 1 \\
\hline \multicolumn{12}{|l|}{ Grupo nigrinus } \\
\hline & P. nigrinus peruvianus & & & & & & & & 1 & & 1 \\
\hline Total* & & $16(3)$ & $5(2)$ & $7(2)$ & $13(4)$ & $10(3)$ & $3(1)$ & $7(2)$ & $1(1)$ & $2(1)$ & 64 \\
\hline
\end{tabular}

1: Colombia, 2: Ecuador, 3: Perú.

*Entre paréntesis se indica el número total de morfotipos recolectados por cuenca.

(Fig. 2E) fueron muy frecuentes en nuestras muestras (17 y 16 ejemplares respectivamente). Mientras $P$. incaicus fue descrita previamente por Brundin (1966) en el altiplano de Bolivia y Perú, ninguna descripción previa se parece a Parochlus sp. 2. Asimismo, cabe destacar a una especie cercana a $P$. ohakunensis (Freeman) (Fig. 2C) (nueve ejemplares recolectados) cuyo borde anterior ondulado del OR es muy característico. Sin embargo, como esta especie solo se ha reportado previamente en Nueva Zelanda (Brundin, 1966), no nos atrevemos a validarla como tal hasta que se pueda confirmar su identidad con posteriores estudios moleculares o con la descripción de los adultos correspondientes.

Dentro del subgrupo araucanus (aquellos con el Tronco del OR alargado) encontramos a Parochlus sp. 1. (Fig. 3C), con un OR muy característico y muy frecuente en nuestros muestreos (17 ejemplares recolectados); mientras que los otros taxones encontrados fueron poco abundantes, incluyendo $P$. nr. maorii (Fig. 3E) que Roback (1970) describió desde Ecuador. Un caso particular es la posible presencia de P. kiefferi (Garrett) en la zona, especie de distribución Holártica. Sólo dos ejemplares examinados procedentes de Colombia y Ecuador se podrían situar como pertenecientes a esta especie, por tener la AS larga, pero con una sola seda y no con dos, como en $P$. araucanus (Fig. 3A) especie que es muy similar. Sin embargo, las sedas de la AS son muy difíciles de distinguir, presentándose una gran variabilidad en su número y posición dentro de una misma especie. Por esta razón además de su baja representatividad, no hemos incluido los especímenes de este morfotipo. Antes de considerar que $P$. kiefferi está presente en nuestra área de estudio, será necesario recolectar más especímenes y probablemente también hacer uso de herramientas moleculares, que permitan confirmar la diagnosis inicial.

Con respecto al género Podonomus, de los 194 individuos examinados, hemos podido distinguir 14 morfotipos en cuatro grupos: decarthrus, nudipennis, albinervis y maculatus (Cuadro 3, Fig. 4, Fig. 5, Fig. 6 y Fig. 7). De éstos, albinervis fue el que presentó más morfotipos (seis), mientras el grupo maculatus 

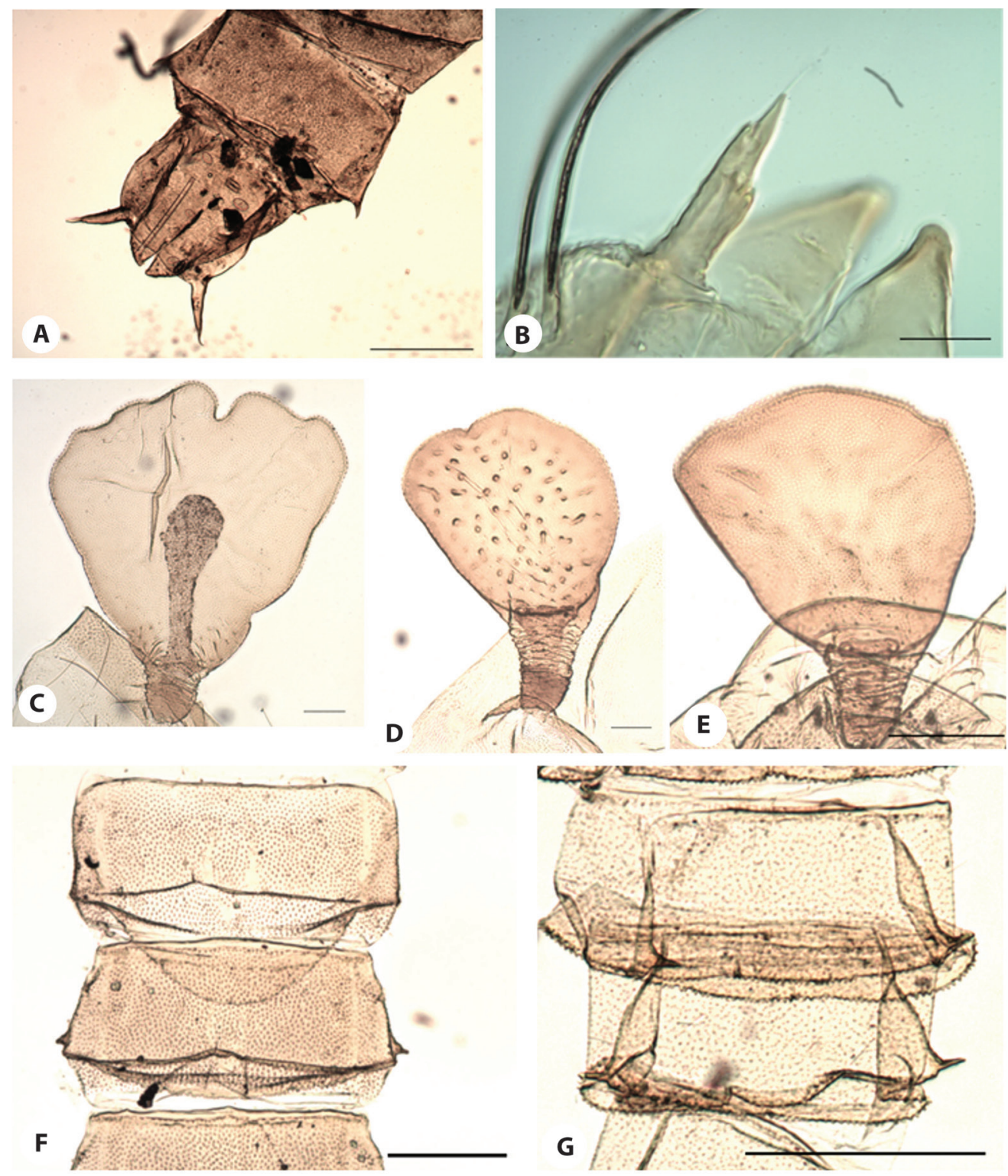

Fig. 2. Parochlus. Segmento Anal y AS: A) P. nigrinus peruvianus; B) $P$. sp. 4. OR: C) $P$. nr. ohakunensis; D) $P$. sp.2; E) $P$. incaicus. Microescultura: F) P. incaicus; G) $P$. sp4. Líneas de escala: $2 \mathrm{~A}(250 \mu \mathrm{m}) ; 2 \mathrm{~B}-2 \mathrm{G}(50 \mu \mathrm{m})$.

Fig. 2. Parochlus. Anal Segment and Anal Spur (AS): A) P. nigrinus peruvianus; B) $P$. sp. 4 . Thoracic horns (TH): C) $P$. nr. ohakunensis; D) P. sp.2; E) P. incaicus. Microsculpture: F) P. incaicus; G) P. sp. 4. Bar scale: $2 \mathrm{~A}(250 \mu \mathrm{m}) ; 2 \mathrm{~B}-2 \mathrm{G}(50 \mu \mathrm{m})$.

solo estuvo representado por $P$. nr. reticulatus (Fig. 6C y 6D). La separación de los grupos de especies de Podonomus es relativamente fácil, primero la mayor o menor extensión de la lámina ventral del segmento abdominal II separa al grupo decarthrus; seguidamente, la forma de los espolones del segmento abdominal VII separa el grupo nudippenis del albinervis. Caso particular es el de $P$. caranqui, que aunque pertenece al grupo albinervis, por motivos prácticos en la clave, se incluye separado de los otros miembros de este grupo, debido a la particular 

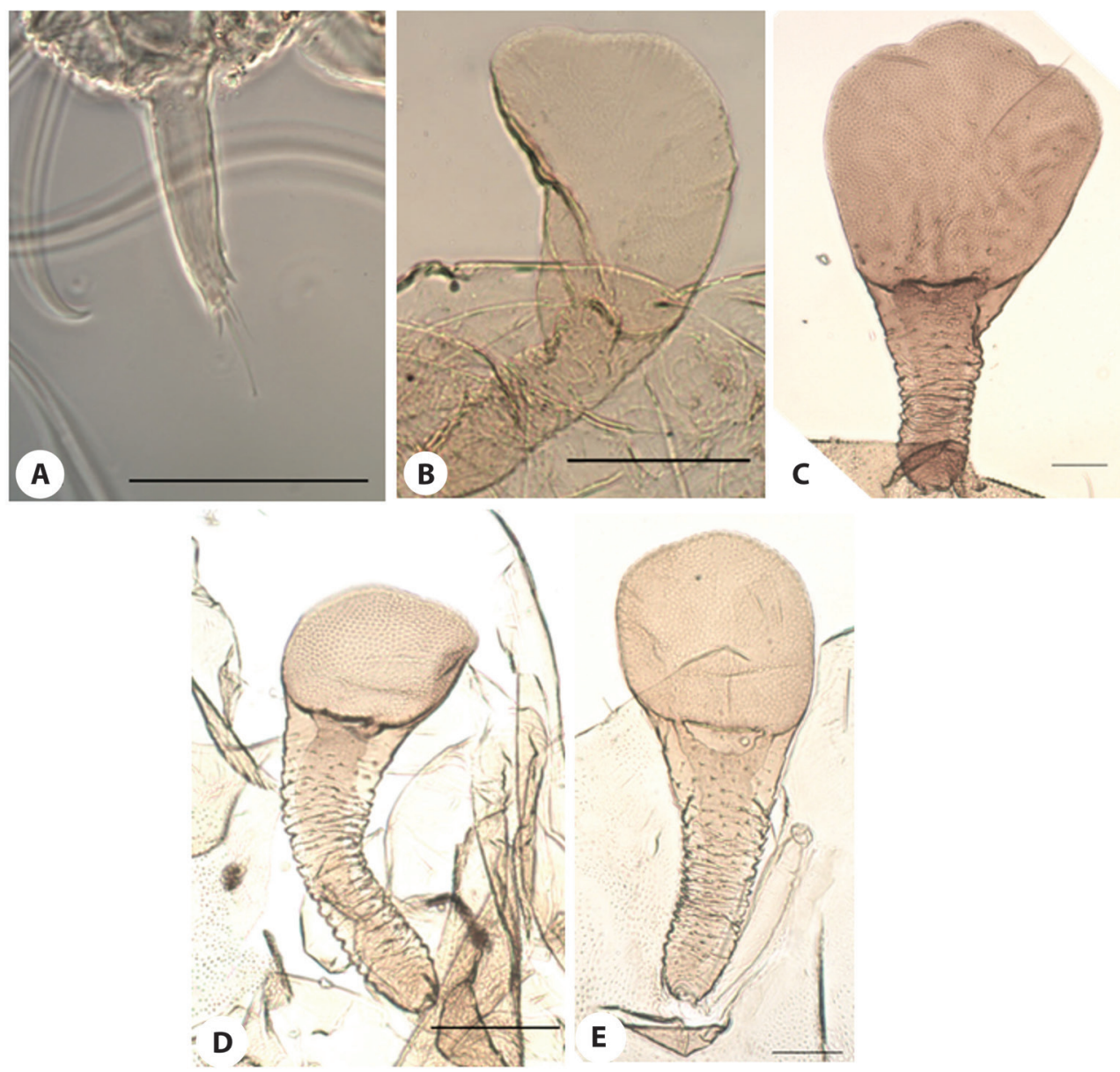

Fig. 3. Parochlus. AS: A) P. nr. araucanus. TH: B) P. nr. araucanus; C) P. sp. 1; D) P. sp. 3; E) P. nr. maorii. Líneas de escala: $3 \mathrm{~A}(25 \mu \mathrm{m})$; 3B-3E $(50 \mu \mathrm{m})$.

Fig. 3. Parochlus. AS: A) P. nr. araucanus. OR: B) P. nr. araucanus; C) P. sp. 1; D) P. sp. 3; E) P. nr. maorii. Bar scale: $3 \mathrm{~A}(25 \mu \mathrm{m}) ; 3 \mathrm{~B}-3 \mathrm{E}(50 \mu \mathrm{m})$.

forma de su OR. Hay que tener precaución con la forma de los espolones, tanto los abdominales como el anal, debido a que en algunas ocasiones pueden estar rotos en el extremo y conducir a identificaciones erróneas.

De los 14 morfotipos de Podonomus encontrados en nuestra zona de muestreo, cuatro fueron reportados previamente, P. fittkaui (Fig. 6F) (grupo decarthrus) y P. fastigians (Fig. 4G y 5A) (grupo nudipennis) fueron descritas por Brundin (1966) en Bolivia y Perú. Así mismo, del grupo albinervis, $P$. nr. regalis fue descrita por Roback y Coffman (1983) en el altiplano de Perú y Bolivia, y de ella hemos distinguido dos formas diferentes según la relación entre la longitud del $\mathrm{T}$ respecto a la PP del OR, ambas recolectadas en Perú. La otra especie previamente descrita, también del grupo albinervis, fue el morfotipo $P$. "La Paz" (Brundin, 1966) que posteriormente Roback (1970) la nombró como P. caranqui (Fig. 4C) a partir de especímenes de Ecuador. Así mismo, encontramos morfotipos similares a las especies originalmente descritas como $P$. illiesi y $P$. reticulatus por Brundin (1966) en la Patagonia chilena, pero debido a su lejanía con nuestra zona de estudio, los hemos nombrado como P. nr. illiesi (Fig. 6G) y P. nr. reticulatus (Fig. 
CUADRO 3

Riqueza y abundancia de especies y morfotipos de pupas y exuvias pupales de Podonomus recolectados en ríos altoandinos

TABLE 3

Richness and abundance of species and morphotypes of pupae and pupal exuviae of Podonomus collected at Andean high altitude rivers

\begin{tabular}{|c|c|c|c|c|c|c|c|c|c|c|c|c|}
\hline & CHIN $^{1}$ & $\mathrm{MIR}^{2}$ & $\mathrm{NAP}^{2}$ & GUAY $^{2}$ & $\mathrm{PAS}^{2}$ & $\mathrm{SAN}^{3}$ & $\mathrm{MOS}^{3}$ & CAÑ ${ }^{3}$ & $\mathrm{COL}^{3}$ & $\mathrm{COT}^{3}$ & $\mathrm{URU}^{3}$ & Total \\
\hline \multicolumn{13}{|l|}{ Grupo decarthrus } \\
\hline P. nr. Illiesi & & & & & & 1 & & 1 & & & & 2 \\
\hline$P$. gr. decarthrus sp. 1 & & & 1 & & & & 1 & & & & & 2 \\
\hline P. gr. decarthrus sp. 2 & & & & 1 & & & & & & 2 & & 3 \\
\hline P. fittkaui & & & & & & 8 & & 21 & 1 & 3 & 1 & 34 \\
\hline \multicolumn{13}{|l|}{ Grupo nudipennis } \\
\hline P. gr. nudipennis sp. 1 & & & & & & & & & 12 & 4 & & 16 \\
\hline P. gr. nudipennis sp. 2 & & 1 & 8 & 1 & & 5 & 4 & 1 & 2 & 1 & & 23 \\
\hline P. fastigians & & & & & & 3 & & 1 & 22 & 1 & & 27 \\
\hline \multicolumn{13}{|l|}{ Grupo albinervis } \\
\hline P. nr. regalis sp. 1 & 2 & & & 1 & & & & 1 & & 3 & & 7 \\
\hline P. nr. regalis sp. 2 & & & & & & & & 5 & & & & 5 \\
\hline P. caranqui & 8 & 2 & & 20 & 1 & 4 & & 4 & & 2 & 1 & 42 \\
\hline P. nr. inermis & & & & & & 12 & & & & 4 & & 16 \\
\hline P. nr. Quito & & & & & & 2 & & 11 & 1 & & & 14 \\
\hline Podonomus sp. 1 & & & & & & 2 & & & & & & 2 \\
\hline \multicolumn{13}{|l|}{ Grupo maculatus } \\
\hline P. nr. reticulatus & & & & & & & & & & 1 & & 1 \\
\hline Total & $10(3)$ & $3(2)$ & $9(2)$ & $23(4)$ & $1(1)$ & $37(8)$ & $5(2)$ & $45(8)$ & $38(5)$ & $21(9)$ & $2(2)$ & 194 \\
\hline
\end{tabular}

1: Colombia, 2: Ecuador, 3: Perú.

*Entre paréntesis se indica el número total de morfotipos recolectados por cuenca.

6C y 6D), respectivamente. Especímenes similares a $P$. quito, especie originalmente descrita en Ecuador por Roback (1970), fueron encontrados en este estudio en las cuencas de Perú, pero debido a algunas diferencias encontradas, preferimos nombrarlos como $P$. nr. quito (Fig. 6B). En cuanto a su frecuencia de ocurrencia, $P$. caranqui, $P$. fittkaui y $P$. fastigians fueron los morfotipos más frecuentes en nuestros muestreos, con 42, 34 y 27 ejemplares recolectados, respectivamente. También fueron relativamente frecuentes Podonomus del grupo nudipennis con dos morfotipos diferentes $(P$. gr. nudipennis sp. 1 y $P$. gr nudipennis sp. 2); hemos encontrado cierta variabilidad en la forma de la PP de estos dos morfotipos. En cuanto a $P$. fastigians, la particular forma de los márgenes posteriores de los tergitos abdominales lo hace inconfundible con otros morfotipos de este grupo. Por el contrario, el único individuo del grupo maculatus, fue encontrado en la cuenca del río Cotahuasi, y presentó la característica ornamentación que describió Brundin (1966) para $P$. reticulatus, pero en la Patagonia de Chile, debido a esto, decidimos nombrar el morfotipo como $P$. nr. reticulatus.

La mayor abundancia de pupas y exuvias de Parochlus, se recolectaron en las cuencas de los ríos Chinchiná (Colombia) y Guayas (Ecuador), con 16 y 13 individuos, respectivamente (Cuadro 2), pero la mayor diversidad de morfotipos se presentó en la cuenca del río Guayas (4 morfotipos). Con respecto a Podonomus, la mayor abundancia se presentó en los ríos de la vertiente del Pacífico de los Andes (Cuadro 3 ), principalmente en las cuencas peruanas de Santa (37 individuos), Cañete (45 individuos) y Colca (38 individuos). Sin embargo, fue la 

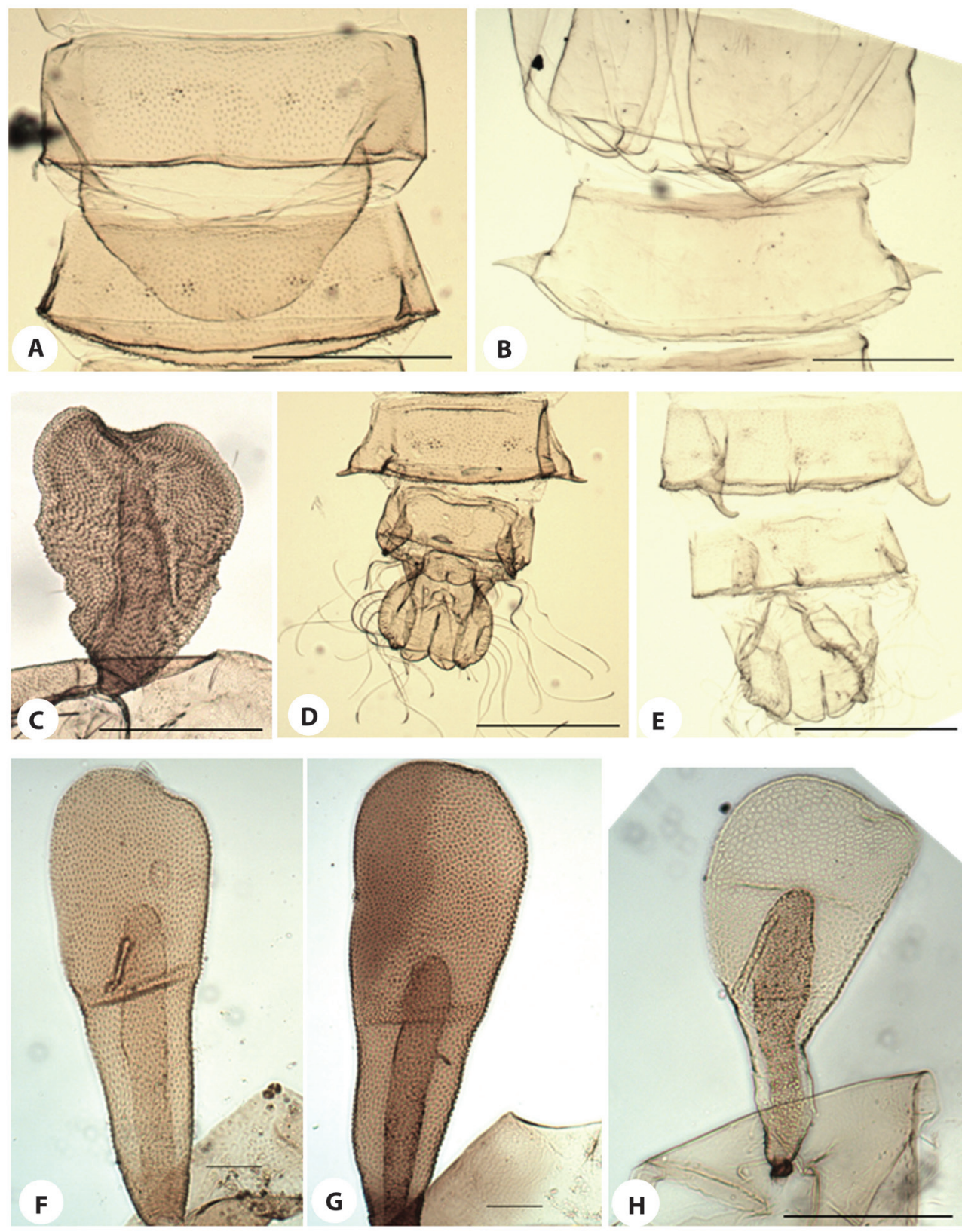

Fig. 4. Podonomus. OR: C) $P$. caranqui; F) $P$ gr nudipennis sp1; G) $P$. fastigians; H) $P$. gr nudipennis sp. 2; Lámina ventral: A) Presente ( $P$. nr. regalis sp. 1); B) Ausente ( $P$. gr. decarthrus sp. 2). Espolones del segmento abdominal VII: D) No recurvados ( $P$. gr. nudipennis sp. 1); E) Recurvados ( $P$. nr. regalis sp. 1). Líneas de escala: 4A-4E, 4H $(250 \mu \mathrm{m}) ; 4 \mathrm{~F}-4 \mathrm{G}$ $(50 \mu \mathrm{m})$.

Fig. 4. Podonomus. TH: C) P. caranqui; F) $P$ gr nudipennis sp. 1; G) P. fastigians; H) P. gr nudipennis sp. 2. Ventral lamella: A) Present ( $P$. nr. regalis sp. 1); B) Absent ( $P$. gr. decarthrus sp. 2). Spurs of the abdominal segment VIII: D) No recurved ( $P$. gr. nudipennis sp. 1); E) Recurved (P. nr. regalis sp. 1). Bar scale: 4A-4E, 4H (250 $\mu \mathrm{m})$; 4F-4G $(50 \mu \mathrm{m})$. 

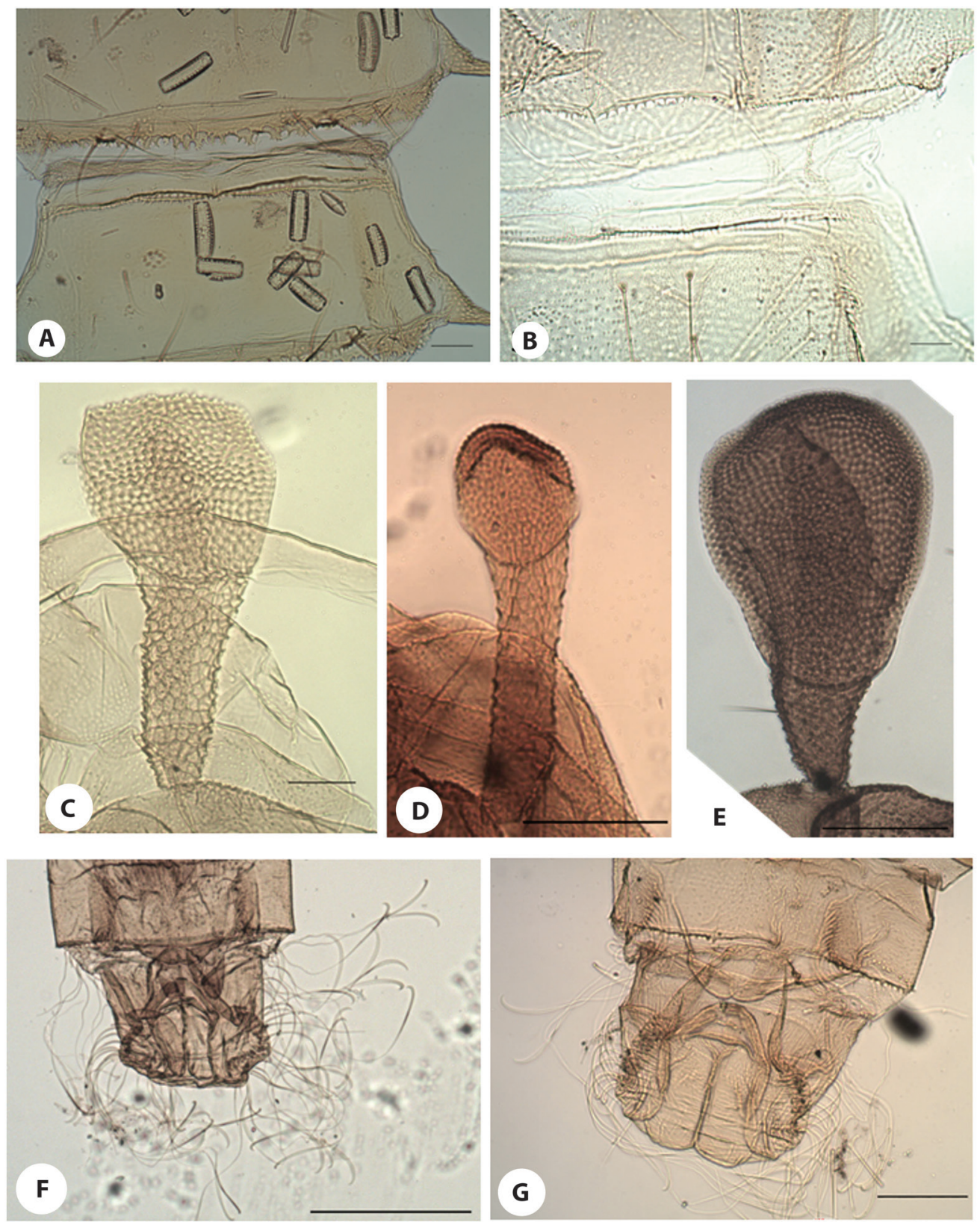

Fig. 5. Podonomus. Tergitos abdominales: A) $P$. fastigians; B) $P$. gr. nudipennis sp. 1. OR: C) $P$. nr. regalis sp. 1; D) $P$. nr. regalis sp. 2; E) $P$. nr. inermis. Sedas onduladas: F) Más de 20 (P. nr. inermis); G) Menos de 15 ( $P$. nr. quito). Líneas de escala: 5A-5D, 5G $(50 \mu \mathrm{m})$; 5E-5F $(250 \mu \mathrm{m})$.

Fig. 5. Podonomus. Abdominal tergites: A) P. fastigians; B) $P$. gr. nudipennis sp. 1. TH: C) $P$. nr. regalis sp. 1; D) $P$. nr. regalis sp. 2; E) P. nr. inermis. Wavy setae: F) More than 20 (P. nr. inermis); G) Less than 15 (P. nr. quito). Bar scale: 5A-5D, 5G $(50 \mu \mathrm{m})$; 5E-5F $(250 \mu \mathrm{m})$. 

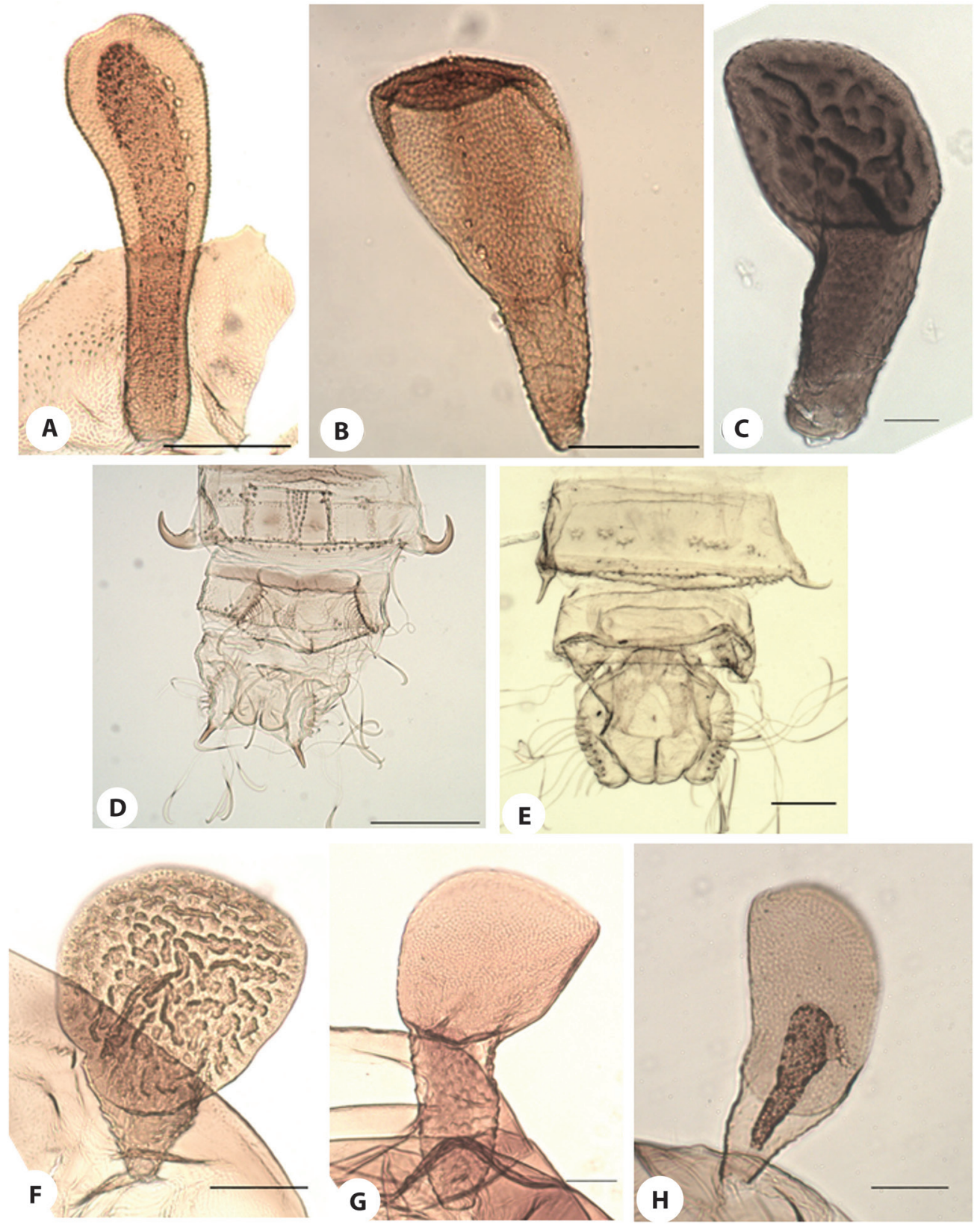

Fig. 6. Podonomus. OR: A) P. sp. 1; B) P. nr quito; C) P. nr. reticulatus; F) P. fittkaui; G) P. nr. illiesi; H) P. gr. decarthrus sp. 1. AS: D) Presente ( $P$. nr. reticulatus); E) Ausente (P. fittkaui). Líneas de escala: 6A-6C, 6E-6H $(50 \mu \mathrm{m}) ; 6 \mathrm{D}(250 \mu \mathrm{m})$. Fig. 6. Podonomus. TH: A) P. sp. 1 ; B) P. nr quito; C) P. nr. reticulatus; F) P. fittkaui; G) $P$. nr. illiesi; H) $P$. gr. decarthrus sp. 1; I) P. gr. decarthrus sp. 1. AS: D) Present (P. nr. reticulatus); E) absent (P. fittkaui). Bar scale: 6A-6C, 6E-6H (50 $\mu \mathrm{m}) ; 6 \mathrm{D}(250 \mu \mathrm{m})$. 

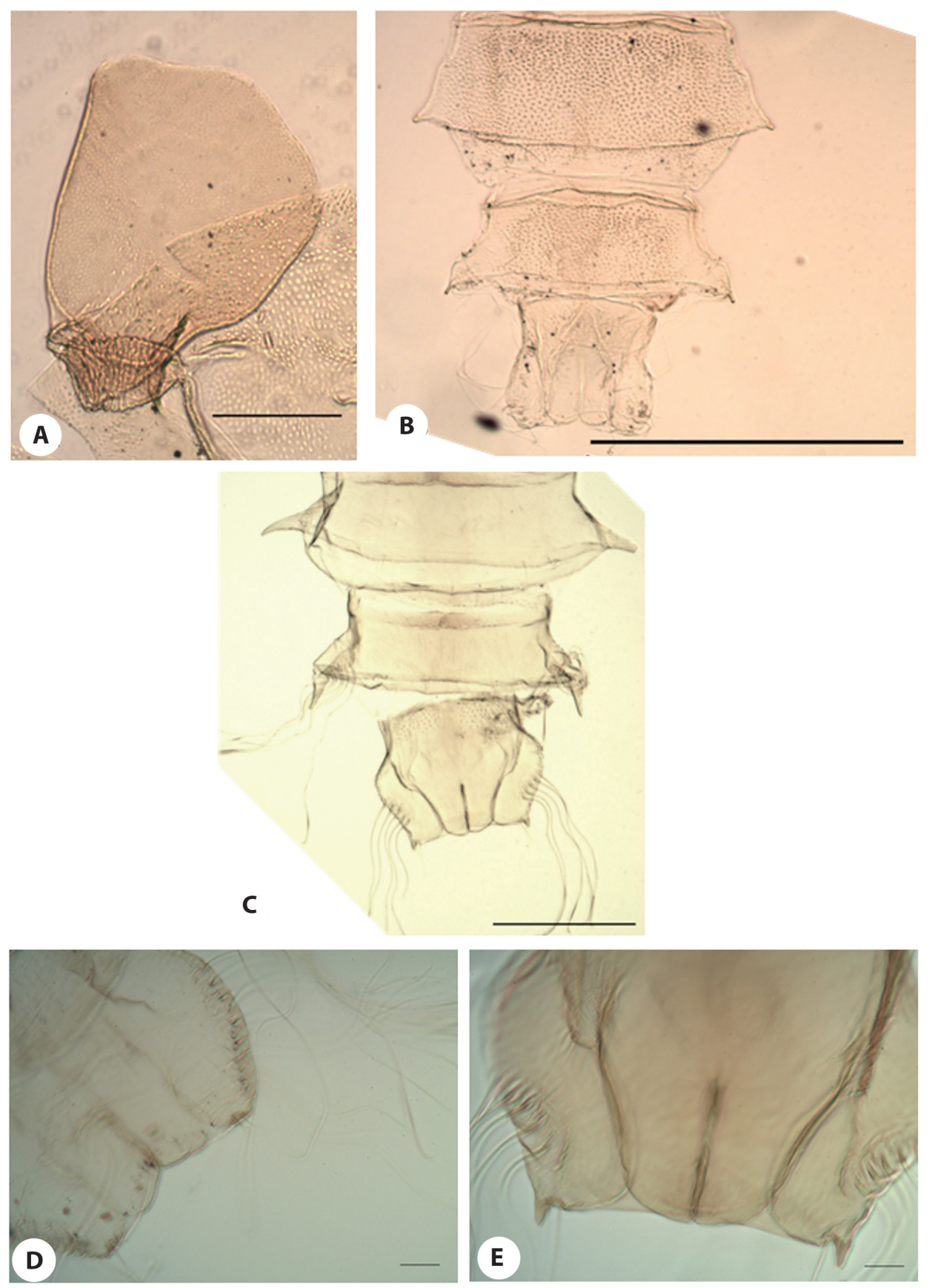

Fig. 7. Podonomus. OR: A) P. gr. decarthrus sp. 2; Espolones del segmento abdominal VIII: B) Cortos (P. nr. illiesi); C) Largos ( $P$. gr. decarthrus sp. 2). AS: D) No evidentes ( $P$. gr. decarthrus sp. 1); E) Cortos ( $P$. gr. decarthrus sp. 2). Líneas de escala: 7A, 7D-7E $(50 \mu \mathrm{m})$; 7B-7C $(250 \mu \mathrm{m})$.

Fig. 7. Podonomus. TH: A) P. gr. decarthrus sp. 2. Spurs of the abdominal segment VIII: B) Short (P. nr. illiesi); C) Long (P. gr. decarthrus sp. 2). AS: D) Inconspicuous ( $P$. gr. decarthrus sp. 1); E) Short ( $P$. gr. decarthrus sp. 2). Bar scale: 7A, 7D-7E $(50 \mu \mathrm{m})$; 7B-7C $(250 \mu \mathrm{m})$. 
cuenca del río Cotahuasi (también de la vertiente del Pacífico), la que presentó la mayor cantidad de morfotipos diferentes recolectados (nueve), seguida de las cuencas de los ríos Santa y Cañete (ocho).

A partir de todos los especímenes examinados, la observación de su morfología y las medidas realizadas, se ha formalizado la siguiente clave, adaptada a partir de Brundin (1966) con la que se pueden distinguir los morfotipos más frecuentes en la zona de estudio. Los acrónimos que se utilizan en la clave son los siguientes: Órgano respiratorio (OR), Longitud del tronco (LT), Longitud de la Placa Porosa (LPP), Anchura de la Placa Porosa (APP), Lámina ventral (LV) y Espolón Anal (AS) (Fig. 1). El Cuadro 4 muestra las principales medidas y relaciones morfométricas (en $\mu \mathrm{m}$ ) para cada morfotipo de Parochlus y Podonomus.

\section{CUADRO 4}

Características morfométricas (medias y rangos) de pupas y exuvias pupales de Parochlus y Podonomus en ríos altoandinos

TABLE 4

Morphometrics characteristics (mean and range) of pupae and pupal exuviae of Parochlus and Podonomus at Andean high altitude rivers

\begin{tabular}{|c|c|c|c|c|c|}
\hline & LT & LPP & APP & LT/LPP & LPP/APP \\
\hline \multicolumn{6}{|l|}{ Parochlus Enderlein, 1912} \\
\hline \multicolumn{6}{|l|}{ Grupo araucanus } \\
\hline P. nr. araucanus & 136.78 & 159.58 & 129.51 & 0.86 & 1.23 \\
\hline Parochlus sp1 & $183.06(151-202)$ & $224.25(179-259)$ & $211.06(178-249)$ & $0.82(0.68-0.94)$ & $1.07(0.95-1.24)$ \\
\hline Parochlus sp3 & 119 & 49 & 6 & 2.43 & 8.17 \\
\hline P. nr. maorii & 197 & 141 & 139 & 1.4 & 1.01 \\
\hline P. nr. ohakunensis & $69.92(55-91.8)$ & $322.64(302-340)$ & $323.08(294-342.55)$ & $0.22(0.18-0.30)$ & $0.99(0.94-1.06)$ \\
\hline P. incaicus & $83.44(68.02-110)$ & $254.91(221-274.78)$ & $248.27(220.83-289)$ & $0.33(0.25-0.50)$ & $1.03(0.91-1.18)$ \\
\hline Parochlus sp2 & $114.92(89-127.5)$ & $261.29(221-307)$ & $270.5(221-329)$ & $0.44(0.35-0.56)$ & $0.97(0.75-1.08)$ \\
\hline Parochlus sp4 & 67.66 & 248.26 & 204.11 & 0.27 & 1.22 \\
\hline \multicolumn{6}{|l|}{ Grupo nigrinus } \\
\hline P. nigrinus peruvianus & 159.67 & 236.57 & 149.43 & 0.67 & 1.58 \\
\hline \multicolumn{6}{|l|}{ Podonomus Philippi, 1865} \\
\hline \multicolumn{6}{|l|}{ Grupo decarthrus } \\
\hline P.nr. Illiesi & $120.31(118-122.62)$ & $163.63(99.26-228)$ & $159.79(111.58-208)$ & $0.88(0.52-1.23)$ & $0.99(0.89-1.10)$ \\
\hline P. gr. decarthrus sp1 & $61.33(45-84)$ & $183.67(145-229)$ & $111.33(88-137)$ & $0.33(0.25-0.37)$ & $1.65(1.62-1.67)$ \\
\hline P. gr. decarthrus sp2 & $179.87(171.60-187)$ & $188(178-207)$ & $168.67(140-198)$ & $0.96(0.9-1.01)$ & $1.13(1.04-1.27)$ \\
\hline P. fittkaui & $71.02(49.15-108.8)$ & $228.489(171.88-393.56)$ & $230.266(175.69-317.46)$ & $0.32(0.12-0.55)$ & $0.99(0.88-1.39)$ \\
\hline \multicolumn{6}{|l|}{ Grupo nudipennis } \\
\hline P. gr. nudipennis sp1 & $63.79(30.52-85)$ & $349.32(234-452.3)$ & $154.4(97.8-232.7)$ & $0.19(0.07-0.30)$ & $2.32(1.45-2.68)$ \\
\hline P. gr. nudipennis sp2 & $84.5(41.36-109.79)$ & $243.52(160.59-224.60)$ & $184.71(120-282.16)$ & $0.37(0.14-0.67)$ & $1.35(1.06-1.67)$ \\
\hline P. fastigians & $51.48(27.69-71.1)$ & $456.56(390.84-523.24)$ & $208.14(155.25-263.44)$ & $0.11(0.05-0.16)$ & $2.21(1.98-2.75)$ \\
\hline \multicolumn{6}{|l|}{ Grupo albinervis } \\
\hline P. nr. regalis $\mathrm{spl}$ & $156.78(124.93-187.24)$ & $151.29(110.88-194.90)$ & $154.4(140-180)$ & $1.09(0.64-1.69)$ & $0.97(0.79-1.08)$ \\
\hline P. nr. regalis sp2 & $195.3(192.19-212.81)$ & $115.25(103.11-127.91)$ & $79.76(64.35-94.06)$ & $1.71(1.53-1.93)$ & $1.47(1.10-1.74)$ \\
\hline P. caranqui & $47.07(29.22-78)$ & $348.09(233.07-416)$ & $271.67(225-343.6)$ & $0.14(0.08-0.29)$ & $1.29(0.73-1.52)$ \\
\hline P. nr. inermis & $118.7(94.9-150.41)$ & $259.57(202.30-323)$ & $180.8(107.4-237.9)$ & $0.46(0.38-0.62)$ & $1.46(1.23-188)$ \\
\hline P. nr. quito & $114.16(97.76-138.21)$ & $216.04(153.73-272.51)$ & $165.81(102-28-207.09)$ & $0.55(0.4-0.82)$ & $1.31(1.1-1.67)$ \\
\hline Podonomus sp1 & $138.5(126-151)$ & $221(182-260)$ & $106.31(106.2-106.42)$ & $0.66(0.48-0.83)$ & $2.08(1.71-2.45)$ \\
\hline \multicolumn{6}{|l|}{ Grupo maculatus } \\
\hline P. nr. reticulatus & 204.9 & 183.6 & 172.2 & 1.12 & 1.07 \\
\hline
\end{tabular}

LT: Longitud del Tronco del Organo Respiratorio, LPP: Longitud de la Placa Porosa del Organo Respiratorio (OR), APP: Ancho de la Placa Porosa del OR. 


\section{Clave para géneros de pupas de la subfamilia Podonominae de los ríos altoandinos (Adaptada a partir de Brundin, 1966)}

1 - Segmentos abdominales III-VII prolongados en procesos laterales en el margen posterior (Fig. 1B y 1C) . . . . . . 2

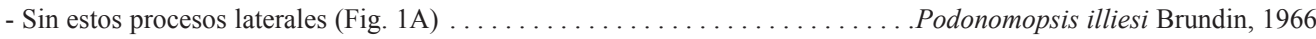

2 - Lóbulos anales como máximo con dos sedas onduladas en cada lado, el mismo segmento tiene dos espuelas anales más o menos desarrolladas que tienen 1-3 sedas muy pequeñas (Fig. 1B) . . . . . . . . . . Parochlus Enderlein, 1912 - Lóbulos anales como mínimo con cinco sedas a cada lado. Si hay espuelas, normalmente están poco desarrolladas y

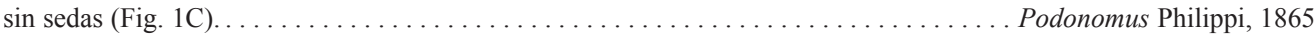

\section{Clave de morfotipos de pupas de Parochlus de los ríos altoandinos (Adaptado a partir de Brundin, 1966)}

1- Sin sedas onduladas en los segmentos abdominales. Espolones anales (AS) presentes y largos, acabados en una seda fuertemente esclerotizada. Sin dentículos en su superficie (Fig. 2A) . . Parochlus nigrinus peruvianus Brundin, 1966 - Con sedas onduladas. Los AS de otra forma con una o dos sedas terminales. . . . . . . . . . . . . .

2- Relación entre la longitud del tronco (LT) del órgano respiratorio (OR) y la longitud de su placa porosa (LPP) inferior

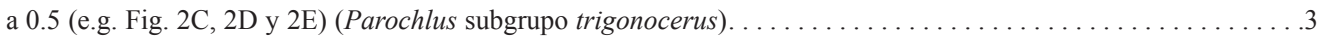

- Relación LT/LPP superior a 0.5 (e.g. Fig. 3C, 3D y 3E) (Parochlus subgrupo araucanus) . . . . . . . . . . .6

3- $\quad$ PP grande y con el borde superior ondulado. LT/LPP media $=0.29(\max =0.5)$ (Fig. 2C).

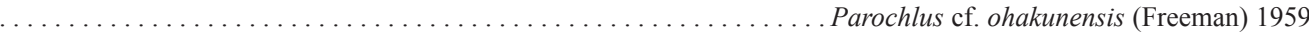

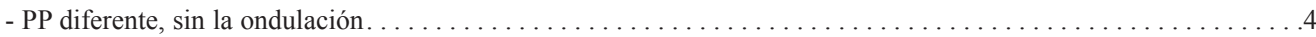

4- Los poros de la PP son muy grandes y distribuidos regularmente por su superficie. Lámina ventral corta (Fig. 2D). La relación LT/LPP media es de 0.44 (rango: 0.35 - 0.56) (Fig. 2D)...................... Parochlus sp. 2 - Los poros de la PP son todos iguales y pequeños. Lámina ventral del segundo segmento abdominal larga (Fig. 2E)5

5- $\quad$ AS cortos con dos sedas cortas. Microescultura del abdomen densa y fuerte (Fig. 2F). Segmento abdominal VIII con

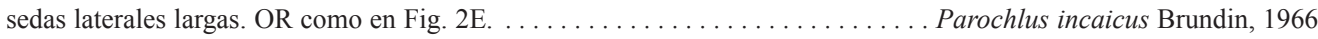
-AS largos (Fig. 2B). Microescultura del abdomen menos densa (Fig. 2G). Segmento abdominal VIII con sedas late-

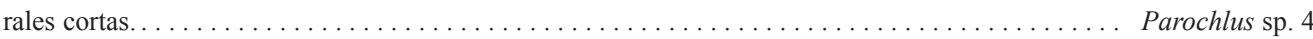

6- $\quad$ AS muy largos, cinco o más veces más largas que anchas (Fig. 3A). LT ligeramente inferior a la LPP (Fig. 3B)

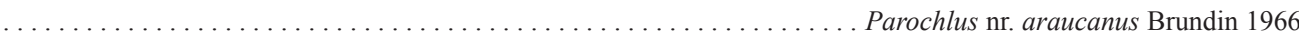

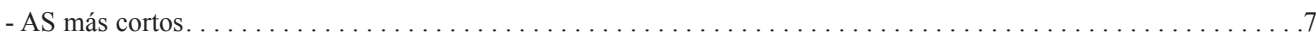

7- Relación LT/LPP inferior a 1, media $=0.82$ (rango: 0.68 - 0.94). Borde de la PP normalmente con tres pequeñas ondu-

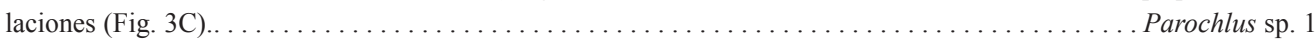

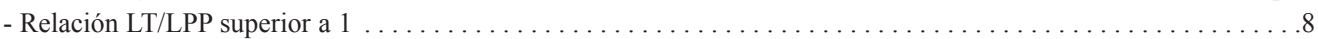

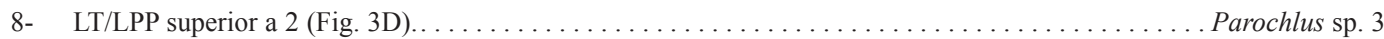
- LT/LPP nunca es superior a 2 (Fig. 3E). AS con dos sedas, la apical más larga que la central . . . . . . . . . . . .

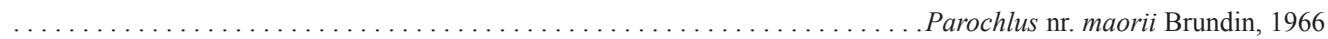

\section{Clave de morfotipos de pupas de Podonomus de los ríos altoandinos (Adaptado a partir de Brundin, 1966)}

1- Lámina ventral (LV) del segmento abdominal II grande, alcanzando más allá de la mitad del tercer segmento abdomi-

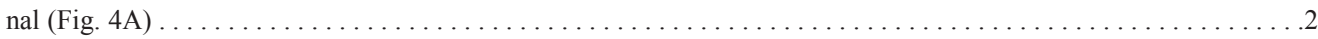
- Lámina ventral más pequeña no sobrepasando la mitad del tercer segmento abdominal (Fig. 4B) . . . . . . . . 10

2- $\quad$ Órgano respiratorio (OR) con el Tronco muy corto y la Placa Porosa (PP) muy ancha. Relación LT/LPP = $0.14(0.08$ - 0.29) y con una escotadura en la parte media de la zona distal muy característica (Fig. 4C).

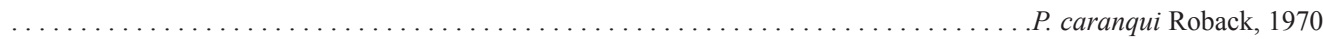

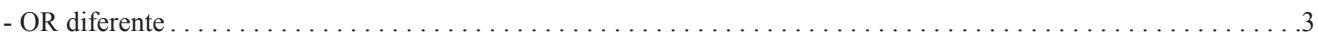

3- Espolones del segmento abdominal VII, dirigidos lateralmente y nunca curvados en la punta (Fig. 4D). El tronco de la

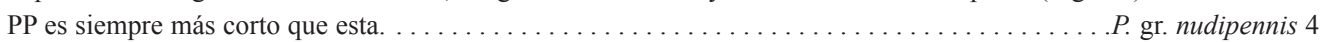
- Espolones del segmento abdominal VII recurvados en la punta (Fig. 4E) $\ldots \ldots \ldots \ldots \ldots \ldots \ldots \ldots \ldots \ldots$ 
4- $\quad$ Tronco del OR extremadamente corto, PP alargada (LT/LPP $=0.14$; rango: $0.05-0.30)$ (Fig. 4F y 4G) . . . . . .5 - Tronco del OR no tan corto (LT/LPP =0.38; rango: 0.24 - 0.67) (Fig. 4H) . . . . . . . . . . gr. nudipennis sp. 2

5- Margen posterior de los tergitos abdominales con dentículos irregulares y ramificados (Fig. 5A) $\ldots \ldots \ldots \ldots \ldots \ldots \ldots \ldots \ldots \ldots \ldots \ldots \ldots \ldots \ldots \ldots \ldots \ldots \ldots \ldots \ldots \ldots \ldots \ldots \ldots \ldots \ldots \ldots \ldots \ldots \ldots$ fastigians Brundin, 1966 Margen posterior de los tergitos abdominales con dentículos cortos no ramificados (Fig. 5B) . .P. gr. nudipennis sp. 1

6- $\quad$ Tronco del OR más largo o igual que la $\mathrm{PP}(\mathrm{LT} / \mathrm{LPP}=1.35$; rango: 0.64 - 1.93) (Fig. 5C y 5D) . . . . P. nr. regalis 7

- Tronco del OR más corto que la PP $(\mathrm{LT} / \mathrm{LPP}=0.53$; rango: $0.38-0.83)($ Fig. $5 \mathrm{E}) \ldots \ldots \ldots \ldots \ldots$

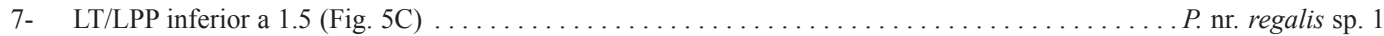

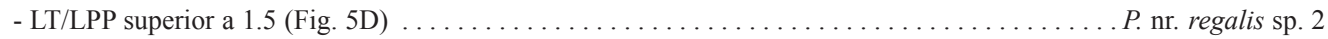

8- $\quad$ Más de 20 sedas onduladas en el segmento anal (Fig. $5 \mathrm{~F}) \ldots \ldots \ldots \ldots \ldots \ldots \ldots \ldots \ldots \ldots \ldots \ldots$ nr. inermis

- Número de sedas onduladas del segmento anal inferior a 15 (Fig. $5 \mathrm{G}) \ldots \ldots \ldots \ldots \ldots \ldots \ldots \ldots \ldots \ldots \ldots$

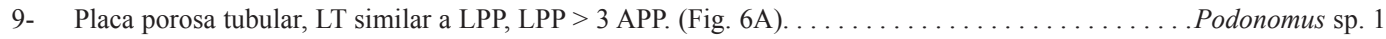

- OR diferente, no tubular LT < LPP, LPP como mucho el doble de APP (Fig. 6B). . . . . . P. nr. quito Roback, 1970

10- Espolones anales (AS) relativamente largos. La ornamentación de los tergitos está formada por una serie de tubérculos

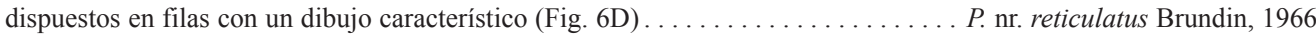

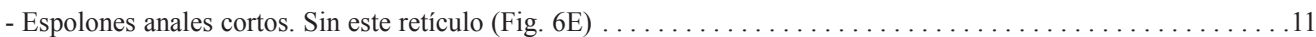

11- Placa porosa de forma redondeada y con poros agrupados en callosidades $(\mathrm{LPP} / \mathrm{APP}=0.99)($ Fig. $6 \mathrm{~F})$ $\ldots \ldots \ldots \ldots \ldots \ldots \ldots \ldots \ldots \ldots \ldots \ldots \ldots \ldots \ldots \ldots \ldots \ldots \ldots \ldots \ldots \ldots \ldots$ fittkaui Brundin, 1966

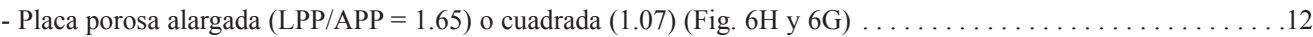

12- Espolones del segmento abdominal VIII cortos y no recurvados (Fig. 7B) $\ldots \ldots \ldots \ldots \ldots \ldots \ldots \ldots \ldots$ P.nr. illiesi Brundin, 1966

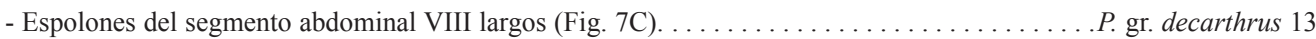

13- OR con el tronco mucho más corto que la PP $(\mathrm{LT} / \mathrm{LPP}=0.33)$ (Fig. 6H). OR más tubular. AS no conspicuos (7D).

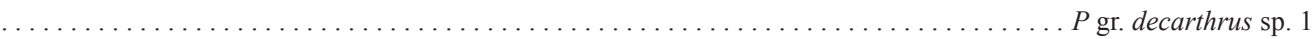
- OR con el tronco más largo, igual o solo algo menor que la PP $($ LT/LPP =0.96) (Fig. 7A). OR más ancho en la parte superior. AS cortos, pero evidentes $(7 \mathrm{E}) \ldots \ldots \ldots \ldots \ldots \ldots \ldots \ldots \ldots \ldots \ldots \ldots \ldots \ldots \ldots \ldots \ldots \ldots \ldots$ gr. decarthrus sp. 2

\section{DISCUSIÓN}

La biodiversidad de las pupas de Podonominae de los ríos altoandinos se ha concretado en la elaboración de una clave taxonómica que permite distinguir los diferentes morfotipos de los tres géneros encontrados, así como dos cuadros que resumen su distribución y frecuencia de ocurrencia. Se encontraron un total de 24 morfotipos de Podonominae, con una sola especie de Podonomopsis (P. illiesi), nueve morfotipos de Parochlus y 14 de Podonomus. Algunos de los morfotipos de Parochlus y Podonomus fueron claramente identificados como especies previamente descritas, tales como Parochlus incaicus, P. nigrinus peruvianus y Podonomus fittkaui, $P$. fastigians y $P$. caranqui; sin embargo, para el resto de morfotipos, estudios posteriores en los que se combine la identificación de pupas, adultos y análisis moleculares, confirmarán si realmente corresponden a especies diferentes.
La distribución geográfica actual de la fauna de Chironomidae en los Andes parece haber sido el resultado conjunto de casos de aislamiento geográfico por el efecto de barreras naturales y severas fluctuaciones climatológicas ocurridas durante el Pleistoceno (Donato \& Posadas, 2010; Prat et al., 2013). En cuanto a la distribución biogeográfica de los Podonominae en los ríos de los Andes, a partir de nuestros muestreos encontramos que, aunque en general tanto Parochlus como Podonomus tienen una buena representación en número de morfotipos en la zona altoandina tropical, esta es menor a la encontrada en los Andes del Sur de Chile y Argentina, con algunos grupos de especies totalmente ausentes en los Andes del Norte y Centro, tales como conjungens, chiloensis, squamipalpis y steineni de Parochlus, por lo que se comprueba la disminución de especies y la simplificación filogenética hacia el norte de la cordillera de los Andes. Sin embargo, este gradiente de menor diversidad 
hacia el norte, no solo es evidente entre los Andes del Centro y del Sur. Más al norte, otros dos patrones de distribución latitudinal son reconocibles. Por una parte, tenemos el caso del género Podonomopsis, cuya única especie en los Andes tropicales ( $P$. illiesi) fue recolectada únicamente en las cabeceras de ríos altoandinos de Perú (preferentemente sobre los $3500 \mathrm{msnm}$ ) y siempre al sur de la Depresión de Huancabamba. Esta depresión geográfica ubicada al norte de Perú en los $6^{\circ}$ de latitud Sur, representa una importante disrupción fisiográfica y estructural en la continuidad de la cordillera de los Andes constituyendo el punto más bajo (2145 msnm) entre Colombia y el sur de Chile (Duellman \& Pramuk, 1999). Las actuales elevaciones y patrones de drenaje en la Depresión de Huancabamba fueron establecidas durante el Pleistoceno periodo durante el cual se sucedieron períodos más fríos y secos durante las fases glaciales intercalados con otros más cálidos y húmedos durante las fases interglaciares (Gansser, 1973). Estas fuertes fluctuaciones climáticas junto con la compleja topografía, presumiblemente resultaron en una barrera natural para la dispersión de diversos grupos taxonómicos y en un área de aislamiento que favoreció altos niveles de endemismos (Duellman \& Pramuk, 1999; Cosacov, Sérsic, Sosa, De-Nova, Nylinder, \& Cocucci, 2009; Weigend, Gottschling, Hilgeri, \& Nürk, 2010). Distribuciones similares se han reportado para otros géneros de Chironomidae como Barbadocladius (Orthocladiinae) con el cual frecuentemente comparte hábitat (Prat et al., 2013). Tanto Barbadocladius como Podonomopsis son géneros de origen gondwánico que en su dispersión hacia el norte no lograron sobrepasar la barrera natural que representa Huancabamba, por lo que están ausentes en los ríos altoandinos de Ecuador y Colombia. Así mismo, las especies de Podonomus, P. fittkaui y $P$. fastigians, muy frecuentes en nuestros muestreos en las cuencas altoandinas de las vertientes del Pacífico de Perú, tampoco fueron encontradas al norte de Huancabamba. En general, se observó tanto una mayor frecuencia de ocurrencia como una mayor diversidad de morfotipos de Podonomus al sur de esta zona.

Por otro lado, tenemos especies de Podonominae que sí han logrado dispersarse al norte de Huancabamba tales como $P$. caranqui y $P$. gr. nudipennis sp. 2 que se han recolectado en todo el gradiente latitudinal estudiado. Otras especies de Chironomidae de origen godwánico también han mostrado dicho patrón, como Stictocladius (Orthocladiinae) (Sæther \& Cranston, 2012; Krosch \& Cranston, 2013). Una posible explicación de porqué algunas especies de Chironomidae consiguieron dispersarse al norte de Huancabamba y otras no, puede estar relacionada con los diferentes requerimientos térmicos a los que algunas especies están adaptadas. Aquellas que lograron dispersarse más al norte, además de sobrellevar la barrera física de Huancabamba, probablemente consiguieron tolerar temperaturas más cálidas, a diferencia de $P$. illiesi, $P$. fittkaui y P. fastigians, especies de requerimientos fríos estenotermos, que quedaron confinadas en las cabeceras de ríos más altos y más fríos, al sur de Huancabamba. Futuros estudios morfológicos y moleculares de larvas y adultos correspondientes a las pupas aquí reconocidas ayudarán a discernir la identidad taxonómica real de los morfotipos descritos en este estudio. Así mismo, es necesaria una mayor recolección de especímenes de Podonominae en otros sectores de los Andes relativamente menos muestreados como en los ríos altoandinos de Bolivia.

\section{AGRADECIMIENTOS}

Este trabajo fue financiado por el Ministerio de Educación y Cultura y Deporte de España mediante los proyectos CGL2005-24168-E; CGL 2006-04333 y CGL2012-16635 y por la Agencia Española de Cooperación Internacional, proyectos: D/011294/07; D/025074/09. A Nuria Sánchez por la preparación de los especímenes. A Iraima Verkaik y Pau Fortuño por la edición de las fotografías. También a los revisores anónimos y a los editores de la revista por sus comentarios y sugerencias. 


\section{RESUMEN}

Podonominae es una subfamilia de Chironomidae cuyos estados inmaduros habitan ríos y arroyos fríos. En América del Sur, la mayoría de las especies han sido reportadas solamente para la subregión Andina/Patagónica. Sin embargo, el conocimiento de su diversidad en áreas tropicales es aún escaso. Estudiamos la riqueza de géneros y morfotipos de pupas y exuvias pupales Podonominae (Diptera: Chironomidae) en los ríos altoandinos de Colombia, Ecuador y Perú y se discutió su distribución geográfica en el área de estudio. Los especímenes fueron recolectados entre 2004 - 2011 en 197 puntos de muestreo, 17 cuencas hidrográficas por encima de los $2000 \mathrm{msnm}$. Durante la estación seca, muestras bentónicas y de deriva fueron tomadas en diferentes sustratos. Reconocimos tres géneros: Podonomopsis, Parochlus y Podonomus, con uno, nueve y 14 morfotipos respectivamente. Sólo seis de ellos fueron claramente determinados como especies previamente descritas. Podonomopsis estuvo representado sólo por una especie, $P$. illiesi, encontrado únicamente en ríos altos de Perú (más de $3500 \mathrm{msnm}$ ). La abundancia y riqueza de morfotipos de Parochlus fueron las altas en ríos de Ecuador y Colombia (P. incaicus, Parochlus sp. 1 y Parochlus sp. 2 fueron las especies más comunes). Por el contrario, Podonomus fue más diverso y abundante en ríos de Perú ( $P$. caranqui, P. fittkaui y $P$. fastigians fueron las especies más comunes). Encontramos un claro patrón de distribución de morfotipos en el gradiente latitudinal. Primero, se confirmó una evidente disminución en la riqueza de morfotipos en relación a los Andes del Sur. Segundo, se encontraron especies como Podomopsis illiesi, Podonomus fittkaui y Podomus fastigians que solamente estuvieron presentes en Perú, pero no en Ecuador y Colombia. Para estas especies, la Depresión de Huancabamba $\left(6^{\circ} \mathrm{S}\right.$ Latitud, Norte de Perú), pudo representar una importante barrera en su dispersión hacia latitudes más bajas. También se presenta una clave taxonómica para la determinación de géneros y morfotipos.

Palabras clave: Chironomidae; América del Sur; macroinvertebrados; insectos acuáticos; ríos altoandinos; exuvias pupales.

\section{REFERENCIAS}

Acosta, R. (2009). Estudio de la cuenca altoandina del rio Cañete (Perú). (Tesis de Doctorado). Universidad de Barcelona, España. Recuperado de: http://www. ub.edu/riosandes/docs/TESIS_RAUL_ACOSTA.pdf

Acosta, R., \& Prat, N. (2010). Chironomid assemblages in high altitude streams of the Andean region of Peru. Fundamental and Applied Limnology, 177(1), 57-79.

Ashe, P., \& O’Connor, J. P. (2009). A World Catalogue of Chironomidae (Diptera). Part 1. Buchonomyiinae, Chilenomyiinae, Podonominae, Aphroteniinae,
Tanypodinae, Usambaromyiinae, Diamesinae, Prodiamesinae and Telmatogetoninae. Dublin: Irish Biogeographical Society \& National Museum of Ireland.

Brundin, L. (1966). Transantarctic relationships and their significance, as evidenced by Chironomid midges, with a monograph of the subfamilies Podonominae and Aphroteniinae and the austral Heptagyidae. Kungl. Svenska Vetenskapsakademiens Handlingar, 1, 1-472.

Cosacov, A., Sérsic, A. N., Sosa, V., De-Nova, J. A., Nylinder, S., \& Cocucci, A. A (2009). New insights into the phylogenetic relationships, character evolution, and phytogeographic patterns of Calceolaria (CALCEOLARIACEAE). American Journal of Botany, 96, 2240-2255.

Cranston, P. S. (2000). The electronic guide of Chironomidae of Australia. Recuperado de: http://apes.skullisland.info/node/3

Cranston P. S., Hardy, N. B., Morse, G. E., Pusledik, L., \& McCluen, S. R. (2010). When molecules and morphology concur: the "Gondwanan" midges (Diptera: Chironomidae). Systematic Ecology, 35, 635-648.

Cranston, P. S., \& Krosh, M. (2015). Evidence from molecules and morphology expands Podonomopsis Brundin (Diptera: Chironomidae: Podonominae) to include 'genus Chile'. Invertebrate Systematics, 29, 610-627.

Cranston, P. S., \& Sæther, O. A. (2010). Australasian Stictocladius Edwards (Diptera: Chironomidae) diagnosed and described. Australian Journal of Entomology, 49, 126-144.

Donato, M., \& Posadas, P. (2010). Biogeographical and ecological comments on "A new Neotropical species of the genus Parochlus Enderlein, 1912 (Chironomidae: Podonominae) and new distribution in Argentina" by Rodríguez et al. 2009. Revista de la Sociedad Entomológica Argentina, 69(1-2), 133-136.

Duellman, W. E., \& Pramuk, J. B. (1999). Frogs of the Genus Eleutherodactylus (Anura: Leptodactylidae) in the Andes of Northern Peru. Scientific Papers. Natural History Museum. The University of Kansas, 13, 1-78.

Epler, J. H. (2001). Identification Manual for the larval Chironomidae (Diptera) of North and South Carolina. A guide to the taxonomy of the midges of the southeastern United States, including Florida. North Carolina: Special Publication SJ2001-SP13.

Gansser, A. (1973). Facts and theories on the Andes. Journal of the Geological Society, 129, 93-131.

Krosch, M., \& Cranston, P. S. (2013). Not drowning, (hand) waving? Molecular phylogenetics, biogeography and evolutionary tempo of the 'Gondwanan' 
midge Stictocladius Edwards (Diptera: Chironomidae). Molecular Phylogenetics and Evolution, 68, 595-603.

Kuhn, J., Andino, P., Calvez, R., Espinosa, R., Hamerlik, L., Vie, S., Dangles, O., \& Jacobsen, D. (2011). Spatial variability in macroinvertebrate assemblages along and among neighbouring equatorial glacier-fed streams. Freshwater Biology, 56(1), 2226-2244.

Pinder, L. C. V., \& Reiss, F. (1986). The pupae of Chironominae (Diptera: Chironomidae) of the Holarctic region - Keys and diagnoses. In Wiederholm T. (ed) Chironomidae of the Holarctic region - Keys and diagnoses. Part 2. Pupae. Entomologica Scandinavica Supplement, 28, 299-456.

Prat, N., González-Trujillo, J. D., \& Ospina-Torres, R. (2014). Clave para la determinación de exuvias pupales de los quironómidos (Diptera: Chironomidae) de ríos altoandinos tropicales. Revista de Biología Tropical, 62(4), 1385-1406.

Prat, N., Ribera, C., Rieradevall, M., Villamarin, C., \& Acosta, R. (2013). Distribution, abundance and molecular analysis of Barbadocladius Cranston \& Krosch (Diptera, Chironomidae) in tropical, high altitude Andean streams and rivers. Neotropical Entomology, 42, 607-617.

Roback, S. S. (1970). Podonominae of Ecuador, with Notes on the Sense Organ and Pupal Respiratory Organs (Diptera, Chironomidae). Journal of the New York Entomological Society, LXXVIII, 148-169.

Roback, S. S., \& Coffman, W. P. (1983). Results of the Catherwood Bolivian-Peruvian Altiplano Expedition. Part II. Aquatic Diptera including montane Diamesinae and Orthocladiinae (Chironomidae) from Venezuela. Proceedings of Natural Sciences of Philadelphia, 135, 9-79.
Roque, F. O., \& Trivinho-Strixino, S. (2004). Podonomus pepinellii $\mathrm{n}$. sp., first record of the genus and subfamily from Brazil (Diptera: Chironomidae: Podonominae). Zootaxa, 689, 1-7.

Sæther, O. A., \& Cranston, P. S. (2012). New World Stictocladius Edwards (Diptera: Chironomidae). Neotropical Entomology, 41, 124-149.

Spies, M., \& Reiss, F. (1996). Catalog and bibliography of Neotropical and Mexican Chironomidae. Spixiana, (Suppl. 22), 61-119.

Thienemann, A. (1937). Podonominae, eine neue Unterfamilie der Chironomiden (Chironomiden aus Lappland I). Miteinem Beitrag: Edwards F. W.: On the European Podonominae (adult stage). Internationale Revue der Gesamten Hydrobiologie, Hydrographie, $35,65-112$.

Trivinho-Strixino, S., Pepinelli, M., Siqueira, T., \& Roque, F. O. (2012). DNA barcoding of Podonomus (Chironomidae, Podonominae) enables stage association of a named species and reveals hidden diversity in Brazilian inselbergs. Annales de Limnologie - International Journal of Limnology, 48, 411-423. doi: 10.1051/limn/2012032

Villamarín, C., Rieradevall, M., Paul, M. J., Barbour, M. T., \& Prat, N. (2013). A tool to assess the ecological condition of tropical high Andean streams in Ecuador and Peru: the IMEERA index. Ecological Indicators, 29, 79-92.

Weigend, M., Gottschling, M., Hilger, H. H., \& Nück, N. M. (2010). Five new species of Lithospermum L. (Boraginaceae tribe Lithospermeae) in Andean South America: Another radiation in the Amotape-Huancabamba Zone. Taxon, 59(4), 1161-1179. 
APÉNDICE 1

Puntos de muestreo de Podonominae (Diptera: Chironomidae) en ríos altoandinos

APPENDIX 1

Sampling sites of Podonominae (Diptera: Chironomidae) at Andean high altitude rivers

\begin{tabular}{|c|c|c|c|c|c|c|c|c|}
\hline $\begin{array}{l}\text { Punto de } \\
\text { muestreo }\end{array}$ & $\begin{array}{l}\text { Fecha de } \\
\text { muestreo }\end{array}$ & País & $\begin{array}{c}\text { Altitud } \\
\text { (m s.n.m.) }\end{array}$ & Latitud & Longitud & $\begin{array}{l}\text { Temperatura } \\
\left(\mathrm{T}^{\circ}\right)\end{array}$ & $\begin{array}{c}\text { Conductividad } \\
(\mu \mathrm{S} / \mathrm{cm})\end{array}$ & Especie/morfotipo \\
\hline CHIN 01 & 14-sep-11 & Colombia & 2341 & $5^{\circ} 03^{\prime} 47^{\prime \prime} \mathrm{N}$ & $75^{\circ} 26^{\prime} 30^{\prime \prime} \mathrm{W}$ & 13.9 & 89.1 & Podonomus nr. regalis sp. 1 \\
\hline CHIN 02 & 14-sep-11 & Colombia & 2485 & $4^{\circ} 59^{\prime} 10^{\prime \prime} \mathrm{N}$ & $75^{\circ} 25^{\prime} 45^{\prime \prime} \mathrm{W}$ & 13.6 & 61.5 & Parochlus sp. 2 (2) \\
\hline CHIN 03 & 15-sep-11 & Colombia & 3408 & $5^{\circ} 06^{\prime} 16^{\prime \prime} \mathrm{N}$ & $75^{\circ} 22^{\prime} 24^{\prime \prime} \mathrm{W}$ & 7.9 & 22.2 & $\begin{array}{l}\text { Podonomus caranqui, } \\
\text { Parochlus sp. } 2 \text { (2) }\end{array}$ \\
\hline CHIN 04 & 15-sep-11 & Colombia & 3433 & $5^{\circ} 06^{\prime} 23^{\prime \prime} \mathrm{N}$ & $75^{\circ} 22^{\prime} 18^{\prime \prime} \mathrm{W}$ & 9.0 & 61.0 & $\begin{array}{l}\text { Podonomus caranqui (5), } \\
\text { Parochlus sp. } 2 \text { (5) }\end{array}$ \\
\hline CHIN 05 & 16-sep-11 & Colombia & 2836 & $5^{\circ} 04^{\prime} 32^{\prime \prime} \mathrm{N}$ & $75^{\circ} 24^{\prime} 33^{\prime \prime} \mathrm{W}$ & 10.6 & 91.6 & $\begin{array}{l}\text { P. caranqui (2), P. nr. regalis sp. } \\
\text { 1, Parochlus. nr. ohakunensis (6), } \\
\text { P. sp3 (1) }\end{array}$ \\
\hline CAMPO 1 & 21-sep-11 & Colombia & 2883 & $4^{\circ} 51^{\prime} 20^{\prime \prime} \mathrm{N}$ & $75^{\circ} 30^{\prime} 38^{\prime \prime} \mathrm{W}$ & 10 & 16.4 & Parochlus nr. kieffieri? \\
\hline MIR 03 & 08-feb-08 & Ecuador & 3078 & $0^{\circ} 11^{\prime} 10^{\prime \prime} \mathrm{N}$ & $78^{\circ} 17^{\prime} 29^{\prime \prime} \mathrm{W}$ & 9.7 & 20.2 & $\begin{array}{l}\text { Parochlus nr. ohakunensis (2), } \\
\text { P. sp2 (2) }\end{array}$ \\
\hline MIR 07 & 09-feb-08 & Ecuador & 2308 & $0^{\circ} 18^{\prime} 37^{\prime \prime} \mathrm{N}$ & $78^{\circ} 14^{\prime} 33^{\prime \prime} \mathrm{W}$ & 17.1 & 437.7 & Podonomus gr. nudipennis sp. 2 \\
\hline MIR 09 & 10 -feb-08 & Ecuador & 3427 & $0^{\circ} 38^{\prime} 45^{\prime \prime} \mathrm{N}$ & $77^{\circ} 53^{\prime} 35^{\prime \prime} \mathrm{W}$ & 9.2 & 26.6 & Parochlus nr. kieffieri? \\
\hline MIR 14 & 12-feb-08 & Ecuador & 3592 & $0^{\circ} 42^{\prime} 27^{\prime \prime} \mathrm{N}$ & $77^{\circ} 58^{\prime} 52^{\prime \prime} \mathrm{W}$ & 7.7 & 39.0 & Parochlus sp. 2 \\
\hline MIR 15 & 13-feb-08 & Ecuador & 3121 & $0^{\circ} 38^{\prime} 30^{\prime \prime} \mathrm{N}$ & $77^{\circ} 56^{\prime} 22^{\prime \prime} \mathrm{W}$ & 12.0 & 71.8 & Podonomus caranqui \\
\hline MIR 16 & 11-feb-08 & Ecuador & 3072 & $0^{\circ} 39^{\prime} 48^{\prime \prime} \mathrm{N}$ & $77^{\circ} 56^{\prime} 51^{\prime \prime} \mathrm{W}$ & 11.7 & 63.5 & Podonomus caranqui \\
\hline GUAY 01 & 14-oct-11 & Ecuador & 3987 & $0^{\circ} 31^{\prime} 50^{\prime \prime} \mathrm{S}$ & $78^{\circ} 13^{\prime} 38^{\prime \prime} \mathrm{W}$ & 9.4 & 102.9 & Podonomus gr. nudipennis sp. 2 \\
\hline GUAY 05 & 22-oct-11 & Ecuador & 3652 & $0^{\circ} 17^{\prime} 14^{\prime \prime} \mathrm{S}$ & $78^{\circ} 14^{\prime} 44^{\prime \prime} \mathrm{W}$ & 10.2 & 68.3 & $\begin{array}{l}\text { Podonomus caranqui (4), } \\
\text { Parochlus sp. } 1 \text { (1), P. nr. maorii }\end{array}$ \\
\hline GUAY 06 & 24-oct-11 & Ecuador & 3468 & $0^{\circ} 27^{\prime} 31^{\prime \prime S}$ & $78^{\circ} 20^{\prime} 00^{\prime \prime} \mathrm{W}$ & 10.0 & 160.9 & $\begin{array}{l}\text { Podonomus caranqui, } P \text {. gr } \\
\text { decarthrus sp. 2, Parochlus sp. } 1 \\
\text { (1), P. Sp. } 2 \text { (4) }\end{array}$ \\
\hline GUAY 07 & 24-oct-11 & Ecuador & 2814 & $0^{\circ} 24^{\prime} 02^{\prime \prime} \mathrm{S}$ & $78^{\circ} 23^{\prime} 02^{\prime \prime} \mathrm{W}$ & 16.2 & 181.5 & $\begin{array}{l}\text { Podonomus caranqui (15), P. nr. } \\
\text { regalis sp. } 1 \text {, Parochlus sp. } 1(4) \text {, } \\
\text { P. sp. } 2 \text { (1), P. nr. ohakunensis (1) }\end{array}$ \\
\hline NAPO 03 & 12-oct-11 & Ecuador & 2672 & $0^{\circ} 22{ }^{\prime} 59^{\prime \prime} \mathrm{S}$ & $78^{\circ} 03^{\prime} 59^{\prime \prime} \mathrm{W}$ & 12.8 & 35.4 & $\begin{array}{l}\text { Podonomus gr decarthrus sp. 1, } \\
\text { Parochlus sp. } 1 \text { (2) }\end{array}$ \\
\hline NAPO 04 & 13-oct-11 & Ecuador & 3712 & $0^{\circ} 23 \prime 20^{\prime \prime} \mathrm{S}$ & $78^{\circ} 12^{\prime} 12^{\prime \prime} \mathrm{W}$ & 8.1 & 241.9 & Podonomus gr. nudipennis sp. 2 (7) \\
\hline NAPO 06 & 13-oct-11 & Ecuador & 3926 & $0^{\circ} 18^{\prime} 05^{\prime \prime} \mathrm{S}$ & $78^{\circ} 07^{\prime} 44^{\prime \prime} \mathrm{W}$ & 12.6 & 40.4 & $\begin{array}{l}\text { Podonomus gr. nudipennis sp. } 2 \text {, } \\
\text { Parochlus incaicus (5) }\end{array}$ \\
\hline PAS 07 & 16-oct-08 & Ecuador & 3274 & $1^{\circ} 25^{\prime} 22^{\prime \prime} \mathrm{S}$ & $78^{\circ} 40^{\prime} 15^{\prime \prime} \mathrm{W}$ & 9.5 & 326.8 & Podonomus caranqui \\
\hline SAN 01 & 15 -oct-07 & Perú & 3324 & $9^{\circ} 31 ' 13^{\prime \prime} \mathrm{S}$ & $77^{\circ} 28^{\prime} 41^{\prime \prime} \mathrm{W}$ & 7.3 & 146.0 & $\begin{array}{l}\text { Podonomus gr. nudipennis sp. } 2 \text { (3), } \\
\text { Podonomus sp. } 1 \text { (2) }\end{array}$ \\
\hline SAN 03 & 15 -oct-07 & Perú & 4039 & $9^{\circ} 31 ' 22 ” \mathrm{~S}$ & $77^{\circ} 38^{\prime} 06^{\prime \prime} \mathrm{W}$ & 13.7 & 198.8 & $\begin{array}{l}\text { Podonomus fittkaui, } \\
\text { P. gr. nudipennis sp. 2, } \\
\text { Podonomopsis illiesi (2) }\end{array}$ \\
\hline SAN 04 & 15 -oct-07 & Perú & 3710 & $9^{\circ} 33^{\prime} 13^{\prime \prime} \mathrm{S}$ & $77^{\circ} 39^{\prime} 37^{\prime \prime} \mathrm{W}$ & 13.3 & 455.0 & Podonomus fastigians (3) \\
\hline SAN 05 & 16 -oct- 07 & Perú & 3364 & $9^{\circ} 22 ’ 57^{\prime \prime} \mathrm{S}$ & $77^{\circ} 31^{\prime} 19^{\prime \prime} \mathrm{W}$ & 7.7 & 27.7 & $\begin{array}{l}\text { Podonomus caranqui (4), } \\
\text { P. nr. inermis, Parochlus incaicus } \\
\text { (3).P. sp. } 1 \text { (5) }\end{array}$ \\
\hline SAN 09 & 18 -oct-07 & Perú & 3859 & $9^{\circ} 03^{\prime} 05^{\prime \prime} \mathrm{S}$ & $77^{\circ} 37^{\prime} 02^{\prime \prime} \mathrm{W}$ & 6.6 & 29.3 & Podonomus nr. Illiesi \\
\hline SAN 11 & 18 -oct-07 & Perú & 4004 & $9^{\circ} 00^{\prime} 41^{\prime \prime} \mathrm{S}$ & $77^{\circ} 41^{\prime} 49^{\prime \prime} \mathrm{W}$ & 7.6 & 21.4 & $\begin{array}{l}\text { Podonomus fittkaui (7), } \\
\text { Parochlus sp. } 1\end{array}$ \\
\hline
\end{tabular}


Apéndice 1 (Continuación) / Appendix 1 (Continued)

\begin{tabular}{|c|c|c|c|c|c|c|c|c|}
\hline $\begin{array}{l}\text { Punto de } \\
\text { muestreo }\end{array}$ & $\begin{array}{l}\text { Fecha de } \\
\text { muestreo }\end{array}$ & País & $\begin{array}{c}\text { Altitud } \\
\text { (m s.n.m.) }\end{array}$ & Latitud & Longitud & $\begin{array}{c}\text { Temperatura } \\
\left(\mathrm{T}^{\circ}\right)\end{array}$ & $\begin{array}{c}\text { Conductividad } \\
(\mu \mathrm{S} / \mathrm{cm})\end{array}$ & Especie/morfotipo \\
\hline SAN 13 & 19-oct-07 & Perú & 3624 & $9^{\circ} 49^{\prime} 26^{\prime \prime} \mathrm{S}$ & $77^{\circ} 25^{\prime} 35^{\prime \prime} \mathrm{W}$ & 12.6 & 225.4 & $\begin{array}{l}\text { Podonomus nr. inermis (11), } \\
\text { P. nr. quito (2), Parochlus sp. } 4\end{array}$ \\
\hline SAN 15 & 19-oct-07 & Perú & 3582 & $9^{\circ} 46^{\prime} 31 " \mathrm{~S}$ & $77^{\circ} 24^{\prime} 54^{\prime \prime} \mathrm{W}$ & 14.3 & 42.1 & Podonomus gr. nudipennis sp. 2 \\
\hline MOS 02 & 3-jul-11 & Perú & 4173 & $9^{\circ} 40^{\prime} 52^{\prime \prime} \mathrm{S}$ & $77^{\circ} 13^{\prime} 36^{\prime \prime} \mathrm{W}$ & 7.9 & 155.8 & $\begin{array}{l}\text { Podonomus gr. nudipennis sp. } 2 \text { (4), } \\
\text { P. gr decarthus sp. } 1 \text { (2) }\end{array}$ \\
\hline MOS 14 & 5-jul-11 & Perú & 3327 & $8^{\circ} 56^{\prime} 08^{\prime \prime} \mathrm{S}$ & $77^{\circ} 22^{\prime} 11^{\prime \prime} \mathrm{W}$ & 10.7 & 105.5 & Parochlus sp. 1 (2) \\
\hline CAÑ 03 & 20 -abr-05 & Perú & 4352 & $12^{\circ} 07^{\prime} 57^{\prime \prime} \mathrm{S}$ & $76^{\circ} 00^{\prime} 39^{\prime \prime} \mathrm{W}$ & 7.0 & 210.0 & Podonomus nr. quito \\
\hline CAÑ 04 & 20 -abr-05 & Perú & 4309 & $12^{\circ} 07^{\prime} 43^{\prime \prime} \mathrm{S}$ & $76^{\circ} 00^{\prime} 44^{\prime \prime} \mathrm{W}$ & 7.0 & 120.0 & Podonomopsis illiesi (2) \\
\hline CAÑ 06 & 20 -abr-05 & Perú & 3913 & $12^{\circ} 05^{\prime} 29^{\prime \prime} \mathrm{S}$ & $75^{\circ} 51^{\prime} 49^{\prime \prime} \mathrm{W}$ & 2.0 & 420.0 & Podonomus fittkaui (2) \\
\hline CAÑ 07 & 20 -abr-05 & Perú & 3935 & $12^{\circ} 05^{\prime} 42^{\prime \prime} \mathrm{S}$ & $75^{\circ} 51^{\prime} 06^{\prime \prime} \mathrm{W}$ & 6.5 & 520.0 & Podonomus fittkaui \\
\hline CAÑ 08 & $20-a b r-05$ & Perú & 4065 & $12^{\circ} 07^{\prime} 57^{\prime \prime} \mathrm{S}$ & $76^{\circ} 00^{\prime} 39^{\prime \prime} \mathrm{W}$ & 2.0 & 590.0 & $\begin{array}{l}\text { Podonomus fittkaui, } \\
\text { Podonomopsis illiesi (10) }\end{array}$ \\
\hline CAÑ 10 & $21-a b r-05$ & Perú & 3906 & $12^{\circ} 06^{\prime} 59^{\prime \prime} \mathrm{S}$ & $75^{\circ} 49^{\prime} 02^{\prime \prime} \mathrm{W}$ & 8.0 & 430.0 & Podonomus fittkaui \\
\hline CAÑ 11 & 21-abr-05 & Perú & 3912 & $12^{\circ} 09^{\prime} 30^{\prime \prime} \mathrm{S}$ & $75^{\circ} 47^{\prime} 50^{\prime \prime} \mathrm{W}$ & 9.0 & 250.0 & $\begin{array}{l}\text { Podonomus nr. quito (2), Parochlus } \\
\text { incaicus, Podonomopsis illiesi (1) }\end{array}$ \\
\hline CAÑ 12 & 21-abr-05 & Perú & 3537 & $12^{\circ} 11^{\prime} 59^{\prime \prime} \mathrm{S}$ & $75^{\circ} 47^{\prime} 50^{\prime \prime} \mathrm{W}$ & 10.0 & 400.0 & Podonomus fittkaui (2) \\
\hline CAÑ 12a & 23-abr-05 & Perú & 3537 & $12^{\circ} 12^{\prime} 30^{\prime \prime} \mathrm{S}$ & $75^{\circ} 47^{\prime} 30^{\prime \prime} \mathrm{W}$ & 10.0 & 400.0 & Podonomus fittkaui (2) \\
\hline CAÑ 13 & 21-abr-05 & Perú & 3450 & $12^{\circ} 14^{\prime} 25^{\prime \prime} \mathrm{S}$ & $75^{\circ} 48^{\prime} 15^{\prime \prime} \mathrm{W}$ & 9.0 & 390.0 & Podonomus fittkaui \\
\hline CAÑ 15 & $21-a b r-05$ & Perú & 4117 & $12^{\circ} 16^{\prime} 16^{\prime \prime} \mathrm{S}$ & $75^{\circ} 52^{\prime} 46^{\prime \prime} \mathrm{W}$ & 4.0 & 50.0 & $\begin{array}{l}\text { Podonomus fittkaui, } P . \mathrm{nr} \text {. regalis } \\
\text { sp. } 1, P . \mathrm{nr} \text {. regalis sp. } 2\end{array}$ \\
\hline CAÑ 16 & $21-a b r-05$ & Perú & 4061 & $12^{\circ} 16^{\prime} 06^{\prime \prime} \mathrm{S}$ & $75^{\circ} 52^{\prime} 39^{\prime \prime} \mathrm{W}$ & 2.5 & 140.0 & $\begin{array}{l}\text { Podonomus caranqui (2), P. nr. } \\
\text { regalis sp. } 2 \text { (2), Parochlus incaicus }\end{array}$ \\
\hline CAÑ 17 & 21-abr-05 & Perú & 3300 & $12^{\circ} 16^{\prime} 45^{\prime \prime} \mathrm{S}$ & $75^{\circ} 49^{\prime} 01 " \mathrm{~W}$ & 8.0 & 320.0 & $\begin{array}{l}\text { Podonomus nr. regalis sp. } 2 \text {, } \\
\text { Parochlus incaicus (2) }\end{array}$ \\
\hline CAÑ 18 & 21-abr-05 & Perú & 3900 & $12^{\circ} 10^{\prime} 33^{\prime \prime} \mathrm{S}$ & $75^{\circ} 44^{\prime} 24^{\prime \prime} \mathrm{W}$ & 9.6 & 300.0 & Podonomus fittkaui (2) \\
\hline CAÑ 19 & 21-abr-05 & Perú & 3900 & $12^{\circ} 10^{\prime} 36^{\prime \prime} \mathrm{S}$ & $75^{\circ} 44^{\prime} 18^{\prime \prime} \mathrm{W}$ & 7.4 & 510.0 & Podonomopsis illiesi (1) \\
\hline CAÑ 20 & 21-abr-05 & Perú & 3850 & $12^{\circ} 10^{\prime} 46^{\prime \prime} \mathrm{S}$ & $75^{\circ} 44^{\prime} 25^{\prime \prime} \mathrm{W}$ & 6.0 & 430.0 & $\begin{array}{l}\text { Podonomus fittkaui (2), } \\
\text { Podonomopsis illiesi (1) }\end{array}$ \\
\hline CAÑ 21 & 23-abr-05 & Perú & 3650 & $12^{\circ} 13^{\prime} 10^{\prime \prime} \mathrm{S}$ & $75^{\circ} 44^{\prime} 15^{\prime \prime} \mathrm{W}$ & 6.5 & 430.0 & Podonomus caranqui (2) \\
\hline CAÑ 22 & 23 -abr- 05 & Perú & 3736 & $12^{\circ} 21^{\prime} 42^{\prime \prime} \mathrm{S}$ & $75^{\circ} 45^{\prime} 48^{\prime \prime} \mathrm{W}$ & 4.0 & 270.0 & $\begin{array}{l}\text { Podonomus fittkaui (3), } \\
\text { Podonomopsis illiesi (1) }\end{array}$ \\
\hline CAÑ 23 & 23 -abr-05 & Perú & 3600 & $12^{\circ} 20^{\prime} 59^{\prime \prime} \mathrm{S}$ & $75^{\circ} 46^{\prime} 32^{\prime \prime} \mathrm{W}$ & 4.4 & 210.0 & Podonomus nr. illiesi \\
\hline CAÑ 26 & 23 -abr- 05 & Perú & 3726 & $12^{\circ} 27^{\prime} 33^{\prime \prime} \mathrm{S}$ & $75^{\circ} 47^{\prime} 51^{\prime \prime} \mathrm{W}$ & 9.4 & 160.0 & $\begin{array}{l}\text { Podonomus gr. nudipennis sp. } 2 \text {, } \\
\text { P. nr. quito (2) }\end{array}$ \\
\hline CAÑ 27 & 23 -abr-05 & Perú & 3350 & $12^{\circ} 27^{\prime} 36^{\prime \prime} \mathrm{S}$ & $75^{\circ} 47^{\prime} 46^{\prime \prime} \mathrm{W}$ & 8.2 & 50.0 & Podonomus fittkaui (2) \\
\hline CAÑ 28 & $23-a b r-05$ & Perú & 3140 & $12^{\circ} 26^{\prime} 17^{\prime \prime} \mathrm{S}$ & $75^{\circ} 49^{\prime} 06^{\prime \prime} \mathrm{W}$ & 8.1 & 90.0 & $\begin{array}{l}\text { Podonomus nr. regalis sp. } 2 \text {, } \\
\text { Parochlus incaicus (2) }\end{array}$ \\
\hline CAÑ 29 & 23 -abr-05 & Perú & 3126 & $12^{\circ} 26^{\prime} 08^{\prime \prime} \mathrm{S}$ & $75^{\circ} 49^{\prime} 57^{\prime \prime} \mathrm{W}$ & 11.2 & 170.0 & Podonomus nr. quito (2) \\
\hline CAÑ 31 & 24-abr-05 & Perú & 2580 & $12^{\circ} 44^{\prime} 39^{\prime \prime} \mathrm{S}$ & $75^{\circ} 49^{\prime} 12^{\prime \prime} \mathrm{W}$ & 9.5 & 140.0 & Podonomus fittkaui \\
\hline CAÑ 33 & 24-abr-05 & Perú & 3208 & $12^{\circ} 48^{\prime} 40^{\prime \prime} \mathrm{S}$ & $75^{\circ} 41^{\prime} 44^{\prime \prime} \mathrm{W}$ & 7.0 & 660.0 & Podonomus fastigians \\
\hline CAÑ 34 & 24-abr-05 & Perú & 2553 & $12^{\circ} 49^{\prime} 42^{\prime \prime} \mathrm{S}$ & $75^{\circ} 44^{\prime} 49^{\prime \prime} \mathrm{W}$ & 12.0 & 550.0 & Podonomus nr. quito \\
\hline CAÑ 35 & 24-abr-05 & Perú & 2563 & $12^{\circ} 50^{\prime} 111^{\prime \prime} \mathrm{S}$ & $75^{\circ} 45^{\prime} 00^{\prime \prime} \mathrm{W}$ & 12.5 & 80.0 & $\begin{array}{l}\text { Podonomus nr. quito (2), Parochlus } \\
\text { nr. araucanus }\end{array}$ \\
\hline COT 01 & 12-jul-11 & Perú & 3278 & $15^{\circ} 07^{\prime} 45^{\prime \prime} \mathrm{S}$ & $72^{\circ} 54^{\prime} 51^{\prime \prime} \mathrm{W}$ & 7.9 & 35.2 & $\begin{array}{l}\text { Podonomus fittkaui (2), P. nr. } \\
\text { regalis sp. } 1 \text { (3) }\end{array}$ \\
\hline COT 02 & 13-jul-11 & Perú & 3470 & $15^{\circ} 03^{\prime} 42^{\prime \prime} \mathrm{S}$ & $72^{\circ} 40^{\prime} 50^{\prime \prime} \mathrm{W}$ & 6.6 & 82.4 & $\begin{array}{l}\text { Podonomus caranqui (2), P. fittkaui, } \\
\text { P. gr. nudipennis sp. } 1 \text { (4), } \\
\text { P. nr. inermis (2), P. nr. reticulatus, } \\
\text { Podonomopsis illiesi (2) }\end{array}$ \\
\hline
\end{tabular}


Apéndice 1 (Continuación) / Appendix 1 (Continued)

\begin{tabular}{|c|c|c|c|c|c|c|c|c|}
\hline $\begin{array}{l}\text { Punto de } \\
\text { muestreo }\end{array}$ & $\begin{array}{l}\text { Fecha de } \\
\text { muestreo }\end{array}$ & País & $\begin{array}{c}\text { Altitud } \\
\text { (m s.n.m.) }\end{array}$ & Latitud & Longitud & $\begin{array}{c}\text { Temperatura } \\
\left(\mathrm{T}^{\circ}\right)\end{array}$ & $\begin{array}{l}\text { Conductividad } \\
(\mu \mathrm{S} / \mathrm{cm})\end{array}$ & Especie/morfotipo \\
\hline COT 03 & 13-jul-11 & Perú & 3854 & $15^{\circ} 01^{\prime} 14^{\prime \prime} \mathrm{S}$ & $72^{\circ} 42^{\prime} 04^{\prime \prime} \mathrm{W}$ & 11.8 & 29.1 & $\begin{array}{l}\text { Podonomus decarthrus sp. } 2 \text { (2), } \\
\text { Podonomopsis illiesi (1) }\end{array}$ \\
\hline COT 04 & 13-jul-11 & Perú & 3640 & $14^{\circ} 59^{\prime} 10^{\prime \prime} \mathrm{S}$ & $72^{\circ} 42^{\prime} 43^{\prime \prime} \mathrm{W}$ & 9.8 & 15 & Podonomopsis illiesi (1) \\
\hline СОТ 05 & 14-jul-11 & Perú & 4486 & $14^{\circ} 54^{\prime} 15^{\prime \prime} \mathrm{S}$ & $72^{\circ} 38^{\prime} 32^{\prime \prime} \mathrm{W}$ & 7.4 & 13.7 & Podonomopsis illiesi (1) \\
\hline СОТ 06 & 14-jul-11 & Perú & 4470 & $14^{\circ} 54^{\prime} 32^{\prime \prime} \mathrm{S}$ & $72^{\circ} 38^{\prime} 18^{\prime \prime} \mathrm{W}$ & 4.6 & 19.2 & Podonomus fastigians \\
\hline СОТ 07 & 14-jul-11 & Perú & 4670 & $14^{\circ} 55^{\prime} 28^{\prime \prime} \mathrm{S}$ & $72^{\circ} 38^{\prime} 20^{\prime \prime} \mathrm{W}$ & 3.6 & 130.7 & $\begin{array}{l}\text { Podonomus gr. nudipennis sp. } 2 \text {, } \\
\text { P. nr. inermis }\end{array}$ \\
\hline COL 01 & 04-oct-08 & Perú & 3735 & $16^{\circ} 14^{\prime} 55^{\prime \prime} \mathrm{S}$ & $71^{\circ} 21^{\prime} 02^{\prime \prime} \mathrm{W}$ & 13.2 & 120.0 & Podonomus fastigians (11) \\
\hline $\mathrm{COL} 02$ & 04-oct-08 & Perú & 2437 & $16^{\circ} 13^{\prime} 54^{\prime \prime} \mathrm{S}$ & $71^{\circ} 42^{\prime} 28^{\prime \prime} \mathrm{W}$ & 18.9 & 444.0 & Podonomus nr. quito \\
\hline COL 05 & 05 -oct-08 & Perú & 4339 & $15^{\circ} 56^{\prime} 01^{\prime \prime} \mathrm{S}$ & $71^{\circ} 22^{\prime} 24^{\prime \prime} \mathrm{W}$ & 10.0 & 46.2 & $\begin{array}{l}\text { Podonomus fastigians (4), P. } \\
\text { gr. nudipennis sp. } 1 \text { (9), P. gr. } \\
\text { nudipennis sp. 2, Parochlus nigrinus }\end{array}$ \\
\hline COL 06 & 05 -oct- 08 & Perú & 4392 & $15^{\circ} 50^{\prime} 09^{\prime \prime} \mathrm{S}$ & $71^{\circ} 28^{\prime} 13^{\prime \prime} \mathrm{W}$ & 17.5 & 123.2 & $\begin{array}{l}\text { Podonomus fastigians (6), } \\
\text { P. gr. nudipennis sp. } 1 \text { (2) }\end{array}$ \\
\hline COL 07 & 05 -oct-08 & Perú & 4800 & $15^{\circ} 45^{\prime} 08^{\prime \prime} \mathrm{S}$ & $71^{\circ} 33^{\prime} 46^{\prime \prime} \mathrm{W}$ & 9.9 & 15.7 & Podonomus fittkaui \\
\hline COL 08 & 05-oct-08 & Perú & 4431 & $15^{\circ} 42^{\prime} 08^{\prime \prime} \mathrm{S}$ & $71^{\circ} 35^{\prime} 36^{\prime \prime} \mathrm{W}$ & 13.2 & 20.3 & $\begin{array}{l}\text { Podonomus gr. nudipennis sp. } 1 \text {, } \\
\text { P. gr. nudipennis sp. } 2\end{array}$ \\
\hline COL 11 & 06-oct-08 & Perú & 3666 & $15^{\circ} 38^{\prime} 38^{\prime \prime} \mathrm{S}$ & $71^{\circ} 36^{\prime} 12^{\prime \prime} \mathrm{W}$ & 15.0 & 267.6 & Podonomus fastigians \\
\hline URU 06 & 09-oct-08 & Perú & 3535 & $13^{\circ} 27^{\prime} 14^{\prime \prime} \mathrm{S}$ & $72^{\circ} 03^{\prime} 40^{\prime \prime} \mathrm{W}$ & 16.6 & 1864.0 & $\begin{array}{l}\text { Podomus fittkaui, } \\
\text { Parochlus incaicus }\end{array}$ \\
\hline URU 09 & 10 -oct- 08 & Perú & 2955 & $13^{\circ} 14^{\prime} 54^{\prime \prime} \mathrm{S}$ & $72^{\circ} 14^{\prime} 37^{\prime \prime} \mathrm{W}$ & 11.8 & 199.6 & Podonomus caranqui \\
\hline
\end{tabular}

*Entre paréntesis se indica el número total de individuos recolectados por morfotipo y punto de muestreo. 